\title{
Low-Thrust Trajectory Design in Low-Energy Regimes using Variational Equations
}

\author{
Rita Neves*1 and Joan-Pau Sánchez ${ }^{2}$ \\ Cranfield University, College Road, Wharley End, Bedford MK43 OAL, United Kingdom
}

\begin{abstract}
This paper proposes a novel description of the equations of motion for lowthrust trajectory design in the presence of a third-body perturbation. The framework is formulated using Gauss' Variational Equations (GVE) with two distinct accelerations: the one produced by the electric engine and the disturbing term of the third-body effect, which is computed using the disturbing potential of the previously studied Keplerian Map. The presented GVE third-body (GVE-3B) framework allows for a simple and intuitive description of the low-thrust optimisation problem. It is accurate until very close to the sphere of influence of the perturbing body, and thus can be used to target trajectories in low-energy regimes. Together with the framework, this paper develops a methodology to generate low-energy first-guess solutions for low-thrust trajectories. Both the methodology and the framework are showcased in the design of two distinct missions: a rendezvous with asteroid 2017 SV19 during its next Earth encounter, after departing from the unstable invariant manifold of the $L_{2}$ point in the Sun-Earth system, and the capture of asteroid 2018 AV2 to a stable invariant manifold of the same point.
\end{abstract}

Keywords: Variational Equations, Low-Thrust, Low-Energy, Trajectory Design, Third-Body Perturbation, Asteroid Missions

\footnotetext{
* Corresponding author

${ }^{1}$ r.neves@cranfield.ac.uk

2jp.sanchez@cranfield.ac.uk
} 


\section{Introduction}

With the pursuit of increasingly innovative and complex space missions, the focus of the space industry has been turning towards low-thrust technologies. Electric propulsion systems provide large savings in propellant mass, which can be decisive for the mission's feasibility: the higher the entire system's mass is, the costlier the endeavour will be, making it less likely to come into fruition. Since the first interplanetary spacecraft using low-thrust was successfully flown in 1998 (Rayman and Lehman, 1997), this technology has allowed for the planning of a range of missions that would otherwise be infeasible, including visits to the outer planets, comets and asteroids (Noton, 2012).

Designing a low-thrust trajectory is a more complex task than doing so for a high-thrust one. For the latter case, the short thrust phases can be approximated by singular events that instantaneously change the spacecraft's velocity. In contrast, low-thrust missions require the propulsion system to operate for a significant part of the transfer, in order to generate the necessary velocity increment - consequently, the thrust vector is a continuous function of time. Thus, the trajectory optimisation problem comprises finding the optimal control law (Dachwald, 2004), i.e. the best sequence of thrust controls required for the spacecraft to meet the mission's objective.

Solving the optimal control problem is an extremely complex task, since no closed-form solutions exist except for some very specific cases (Ross and Fahroo, 2002). The problem's convergence depends on several different factors. One of them is the definition of bounds, boundary conditions and constraints for the trajectory. Another is the choice of model of motion in which the trajectory is developed (Shirazi et al., 2018).

Many authors have selected Gauss' Variational Equations (GVE) to solve the optimal control problem, either in their classical or averaged form (Sánchez and Yárnoz, 2016; Herman and Spencer, 2002; Geffroy and Epenoy, 1997; Gao, 2007). This formulation models the motion of the spacecraft subject to a central gravitational force in the two-body problem (2BP) and an additional perturbing effect-for instance, the propulsive acceleration from the spacecraft's engine. It is a common choice for trajectory design due to the model's presentation in Keplerian elements, which provide an intuitive perception of the evolution of the motion (Shirazi et al., 2018). Given that the solution to an optimal control problem typically relies on the setting of parameters by an expert in astrodynamics, outside of the actual optimisation 
process (Dachwald, 2004), it is crucial to have an intuitive understanding of how the trajectory unfolds until a solution is reached.

However, certain design applications, like missions to near-Earth asteroids (NEAs) require models of motion of higher complexity than the classical 2BP. In particular, third-body perturbations may have a non-negligible effect, since many accessible NEAs (Yárnoz et al., 2013) move in low-energy regimes. These are here defined as regimes of motion in which ballistic capture is theoretically possible (Koon et al., 2001). This may occur for objects whose orbital energy does not differ much from that of the third-body perturbation, e.g. nearly co-orbital bodies (Nesvorny et al., 2007) or, in the case of the Sun-Earth system, spacecraft departing from or arriving to Earth with a low excess velocity $\left(v_{\infty}\right)$. However, the utilisation of an alternative higher-fidelity method (e.g. the circular restricted three-body problem (CR3BP)) may bring difficulties related to the definition of the optimal control problem - for instance, boundary conditions are not trivial to set, since the coordinates are presented in the synodic Cartesian reference frame. Instead, using the Keplerian element formulation provided by the GVE, the bounds, boundary values and trajectory evolution of the optimal control problem can be more easily assessed, facilitating its formulation and the convergence to obtain a control law solution.

Thus, this paper proposes a new set of equations - referred here as the GVE third-body (GVE-3B) framework - to model third-body motion together with the acceleration of a low-thrust engine. These are obtained by propagating the classical GVE with the sum of two disturbing acceleration vectors. The first is obtained from the thrust produced by the electric engine; the second corresponds to the third-body perturbation, derived from the Keplerian Third-Body Potential function (K3BP) of the Keplerian Map $(\mathrm{KM})$. The latter is a method to compute the change of the orbital elements throughout one period of the motion, as caused by the third-body effect. Its development started with the works of Petrosky and Broucke (1987) and of Chirikov and Vecheslavov (1993); the concept was then continued by Ross and Scheeres (2007) to study distant flybys in the planar CR3BP. Later on, it was expanded by Alessi and Sánchez (2015) for three-dimensional applications.

In order to test the capabilities of the GVE-3B, this formulation is used to design trajectories in the low-energy regime - namely, low-thrust missions to NEAs. Asteroids have been at the forefront of space exploration for many years, with the proposal and completion of missions such as JAXA's 
Hayabusa (Kawaguchi et al., 2008) and NASA's Dawn (Russell and Raymond, 2011). There are many reasons for the current interest in these bodies: from the fact that their study may answer questions about the formation and evolution of the Solar System (Glassmeier et al., 2007), to their abundance in potentially valuable resources and materials for space manufacturing (Lewis and Hutson, 1993; Elvis, 2012). Furthermore, although main-belt asteroids are known since the $19^{\text {th }}$ century, the discovery of NEAs only happened much later. These asteroids are now considered the easiest celestial bodies to reach from the Earth and, in consequence, they may represent a potential impact threat to our planet (Alvarez et al., 1980).

Particularly, two specific trajectories in the low-energy regime are here designed: an asteroid rendezvous and a capture mission. The first case consists on a spacecraft departing the $L_{2}$ point of the Sun-Earth system through the unstable invariant manifold orbit and matching its motion to the asteroid's. The second entails having a spacecraft attaching itself to another asteroid and moving it, from its nominal orbit to the stable invariant manifold of the same point.

The main goal of this paper is to contrast the GVE-3B with the commonly used 2BP formulation, which models only the single gravitational force of a central body, together with the low-thrust acceleration of the spacecraft. The model comparison uses the CR3BP as the baseline with which the propagation errors are computed. It is postulated that the inclusion of the third-body effects during the design of the controls for a low thrust transfer will yield much higher accuracies than with the standard 2BP. Ultimately, this accuracy is necessary in order to reap the benefits of low energy transfers.

In addition to this, a methodology to develop first-guess low-thrust trajectories is presented. Solving the optimal control problem to design low-thrust trajectories is mostly done using direct methods (Shirazi et al., 2018; Betts, 1998), and these generally require the generation of a reasonable initial guess of the solution parameters (Conway, 2010), i.e. a set of state and control time-histories that yield a sub-optimal trajectory to be improved by the NLP problem solver (in this paper, GPOPS-II (Patterson and Rao, 2014)). The computation of first-guess trajectory is here achieved by a sequential approach, from an impulsive guess to a multiple shooting correction of the motion.

This paper is organised in the following manner: Section 2 provides the necessary background on the classical models used in this paper: the 2BP, the CR3BP and its related dynamical structures. Section 3 sets up the GVE- 
3B framework, demonstrates the computation of the disturbing accelerations and provides an overview of the method's accuracy. Section 4 details the steps involved in the trajectory design for the capture and rendezvous missions, until the full low-thrust transfer is obtained. Section 5 presents the results of the full trajectory designs and compares the obtained control laws in the previously discussed models. Finally, Section 6 enumerates some recommendations and future working areas of interest.

\section{Models of Motion}

\subsection{The Two-Body Problem}

The mathematical problem of designing a trajectory in the Solar System, an environment composed of countless bodies, is named the $n$-body problem, where $n$ stands for the amount of bodies interacting with the spacecraft. The $2 \mathrm{BP}$ is the case when $n=2$ : in order to compute it, Eq. (1) is used (Ulrich, 2019):

$$
\ddot{\boldsymbol{r}}=-\mu_{C} \cdot \frac{\boldsymbol{r}}{\|\boldsymbol{r}\|^{3}}
$$

where $\boldsymbol{r}$ is the position vector relative to the central body, and $\mu_{C}=G m_{C}$ is the gravitational parameter of the central body, equal to the product of the gravitational constant $G$ and the object's mass.

\subsection{Circular Restricted Three-Body Problem}

When computing trajectories in the Solar System, the complexity of the model of motion increases with the number of bodies interacting. The motion of any system with more than two objects has no general analytical solutions. Consequently, the main strategy to model movement in a threebody system is the CR3BP, a simplification of the three-body problem whose equations are solved using a numerical integrator. In the CR3BP, the computed body's mass is deemed insignificant compared to the other two, which move in circular orbits around each other. The former body is regularly termed the massless particle, while the non-massless ones are named the primaries - alternatively, primary is used for the bigger body, while secondary is employed for the smaller of the two. 


\subsubsection{Equations of Motion}

The CR3BP is typically represented in the synodic reference frame; all the physical quantities are normalised in such a way that both the sum of the primaries' mass and the distance that separates them is equal to 1 (Szebehely, 1967). The normalised position and velocity for the massless particle in the CR3BP obey the following equations of motion:

$$
\begin{aligned}
\ddot{x}-2 \dot{y} & =-\frac{\partial \bar{U}}{\partial x} \\
\ddot{y}+2 \dot{x} & =-\frac{\partial \bar{U}}{\partial y} \\
\ddot{z} & =-\frac{\partial \bar{U}}{\partial z}
\end{aligned}
$$

where $\bar{U}$ is the effective potential function of the system:

$$
\bar{U}=-\frac{1}{2}\left(x^{2}+y^{2}\right)-\frac{1-\mu}{r_{1}}-\frac{\mu}{r_{2}}
$$

in which $r_{1}=\left\|\boldsymbol{r}_{1}\right\|$ and $r_{2}=\left\|\boldsymbol{r}_{2}\right\|$ are the distances from the massless particle to the primary and the secondary, respectively. The normalised gravitational parameter $\mu$ is determined as $\frac{\mu_{2}}{\mu_{1}+\mu_{2}}$, in which $\mu_{1}$ and $\mu_{2}$ are the gravitational parameters of the primary and secondary.

\subsubsection{Dynamical Structures}

The equations of motion of the CR3BP admit five equilibrium solutions, known as the libration points $L_{i}, i=1,2, \ldots 5$. In their vicinity, one can find periodic orbits, also called libration point orbits (LPO): these are characterized by a motion that repeats itself after a time period, as seen relative to the synodic reference frame.

Given that the LPO of the Sun-Earth system are not stable, one can find hyperbolic invariant manifold structures connected to them (Gomez et al., 2001). Geometrically, these are dynamical structures composed of countless orbits. They exist for a range of energies and form a series of 'tubes' that connect different regions around the primaries. The unstable manifold is formed by an infinite set of trajectories that exponentially leaves the LPO. On the other hand, the stable manifold consists of an infinite number of trajectories exponentially approaching it.

These manifold orbits can be used to explore new spacecraft trajectories with interesting characteristics (Koon et al., 2000): by moving a body to an 
invariant manifold trajectory connected to an LPO, it will arrive to the LPO without any further manoeuvring (Koon et al., 2008). Since their orbits can be travelled by a spacecraft without any fuel consumption, the exploration of these dynamical structures is particularly interesting for mission design.

\section{Gauss' Variational Equations' Framework}

\subsection{Equations of Motion}

The GVE have been extensively used in astrodynamics to compute motion perturbed by a disturbing acceleration. Following Battin (Battin, 1999), the equations can be formulated in this manner:

$$
\begin{aligned}
\frac{d a}{d t} & =\frac{2 a^{2}}{L}\left(a_{r} e \sin \nu+a_{\theta} \frac{p}{r}\right) \\
\frac{d e}{d t} & =\frac{1}{L}\left(a_{r} p \sin \nu+a_{\theta}((p+r) \cos \nu+r e)\right) \\
\frac{d i}{d t} & =a_{h} \frac{r \cos v}{L} \\
\frac{d \Omega}{d t} & =a_{h} \frac{r \sin v}{L \sin i} \\
\frac{d \omega}{d t} & =\frac{1}{L e}\left(-a_{r} p \cos \nu+a_{\theta}(p+r) \sin \nu\right)-a_{h} \frac{r \sin v \cos i}{L \sin i} \\
\frac{d \nu}{d t} & =\frac{L}{r^{2}}+\frac{1}{L e}\left(a_{r} p \cos \nu-a_{\theta}(p+r) \sin \nu\right)
\end{aligned}
$$

where the set $\{a, e, i, \Omega, \omega, \nu\}$ corresponds to the commonly known orbital elements with $\nu$ as the true anomaly; $a_{r}, a_{\theta}$ and $a_{h}$ are the acceleration components in the Local Vertical, Local Horizontal (LVLH) frame, $L$ is the angular momentum, $p$ is the semilatus rectum, $r$ is the orbital position and $v=\nu+\omega$ is the argument of latitude.

In contrast to Lagrange Planetary Equations (Battin, 1999), GVE can account for both conservative and non-conservative accelerations. In the case of the GVE-3B framework, these consist of the sum of the third-body effect with the low-thrust acceleration of the spacecraft. Without taking into account the different frame of reference changes, the GVE-3B acceleration can be described by Eq. (5).

$$
\boldsymbol{a}_{G V E}=\left\{a_{r}, a_{\theta}, a_{h}\right\}=\boldsymbol{a}_{L T}+\boldsymbol{a}_{3 B}
$$


According to Newton's first law, $\boldsymbol{a}_{L T}$ is easily computed as the quotient of the thrust vector and the system's mass. The formulation of $\boldsymbol{a}_{3 B}$ is based on the disturbing function of the KM method (Ross and Scheeres, 2007; Alessi and Sánchez, 2015). This model is valid for planetary systems where the normalised gravitational parameter is very small - the case of the Sun-Earth system, which will be here taken as the main example.

\subsection{Disturbing Accelerations}

The three-body planetary system from which the disturbing potential function is derived can be seen on Figure 1. Two reference frames can be inferred from the picture. The first is the regular inertial Cartesian frame, centred on the Sun and represented by $O_{\text {Ixyz }}$. The second is a novel one named the Earth-pointing reference frame, denoted by $O_{\oplus x y z}$, with its origin on the barycentre. The definition of this new frame of reference is required for the computation of the disturbing function, which is used to derive the GVE-3B acceleration.

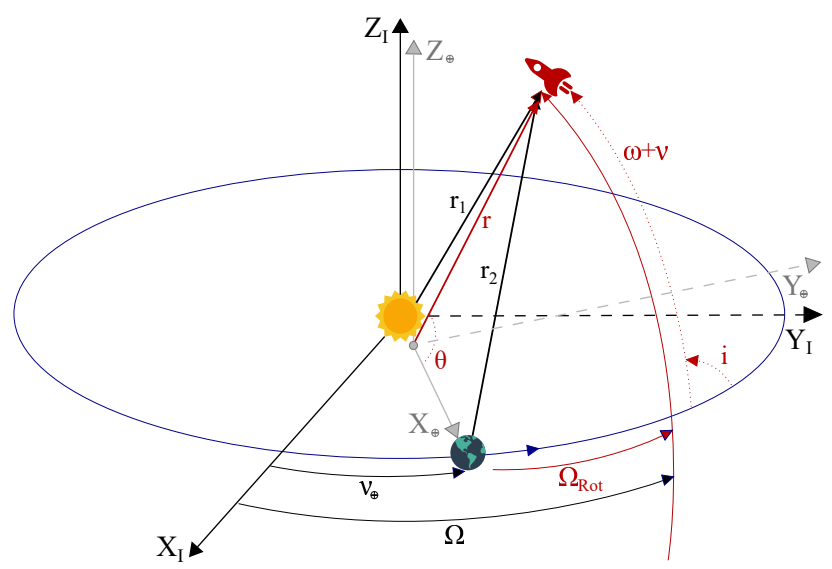

Figure 1: Three-dimensional geometry of the three-body problem, noting the inertial and Earth-pointing reference frames (Neves and Sánchez, 2018)

Thus, a new element of motion can be observed: $\Omega_{R o t}$, which is the rotational longitude of the ascending node of the spacecraft. It is defined in such a way that the new frame's X-axis is always aligned with the Earth (Alessi and Sánchez, 2015). As such, the movement in the Earth-pointing reference frame is instantaneously inertial, barycentric, measured from the Earth axis. This $\Omega_{R o t}$ replaces the regular $\Omega$ in Eqs. (4); it is computed using the true 
anomaly of the Earth, and the longitude of the ascending node $\Omega$ :

$$
\Omega_{R o t}=\Omega-\nu_{\oplus}
$$

Following Alessi and Sánchez (2015), $\nu_{\oplus}$ can be formulated as a function of the orbital elements of the spacecraft, as in Eq. (7). The orbital elements of the latter are represented without any subscript, in contrast to the orbital elements of the Earth (subscript $\oplus$ ). The full derivation of this relationship can be found in (Neves, 2019):

$$
\nu_{\oplus}-\nu_{\oplus_{0}}=\sqrt{\frac{a^{3}}{1-\mu}} M+t_{0}
$$

in which $M$ is the mean anomaly of the spacecraft.

Thus, the change to the Earth-pointing reference frame can be used to describe the third-body perturbation without having to explicitly compute the position of the secondary at all times. However, this comes at the cost of having a non-autonomous reference frame.

After setting up the desired reference frame, the computation of the disturbing accelerations for Eqs. (4) requires the Hamiltonian of the three-body problem in an inertial reference frame, Sun-centred:

$$
\mathcal{H}_{3 B}=\frac{1}{2}\left(o_{x}^{2}+o_{y}^{2}+o_{z}^{2}\right)-\frac{1-\mu}{r_{1}}-\frac{\mu}{r_{2}}
$$

in which $o_{x}, o_{y}$, and $o_{z}$ are the generalized momenta of the massless particle. In order to reach a barycentric notation, $r_{1}$ and $r_{2}$ have to be written as functions of the distance to the barycentre $r$. These two variables are described by the following equations:

$$
\begin{aligned}
& r_{1}^{2}=(x+\mu)^{2}+y^{2}+z^{2} \\
& r_{2}^{2}=(x-1+\mu)^{2}+y^{2}+z^{2}
\end{aligned}
$$

By manipulating Eqs. (10), using polar coordinates and assuming a Taylor expansion around $\mu=0$, Eqs. (11) and (12) are obtained.

$$
\begin{aligned}
\frac{1-\mu}{r_{1}} & \approx \frac{1}{r}+\mu\left(-\frac{1}{r}-\frac{\cos \theta}{r^{2}}\right)+\mathcal{O}\left(\mu^{2}\right) \\
\frac{\mu}{r_{2}} & \approx \frac{\mu}{\sqrt{r^{2}-2 r \cos \theta+1}}+\mathcal{O}\left(\mu^{2}\right)
\end{aligned}
$$


Thus, Eq. (8) can be written in the following form:

$$
\begin{aligned}
\mathcal{H}_{3 B} & =\mathcal{T}+\mathcal{U}_{3 B}+\mathcal{O}\left(\mu^{2}\right) \\
\mathcal{T} & =\frac{1}{2}\left(o_{x}^{2}+o_{y}^{2}+o_{z}^{2}\right)-\frac{1}{r} \\
\mathcal{U}_{3 B} & =\mu\left(\frac{1}{r}+\frac{\cos \theta}{r^{2}}-\frac{1}{\sqrt{1+r^{2}-2 r \cos \theta}}\right)
\end{aligned}
$$

where the physical quantities $r$ and $\cos \theta$ are simply defined as Eq. (16), following the geometry of Figure 1:

$$
r=\sqrt{x^{2}+y^{2}+z^{2}}, \quad \cos \theta=\frac{x}{r}
$$

The accelerations relative to the third-body effect only are computed using Eq. (15):

$$
a_{x}=-\frac{\partial \mathcal{U}_{3 B}}{\partial x}, a_{y}=-\frac{\partial \mathcal{U}_{3 B}}{\partial y}, a_{z}=-\frac{\partial \mathcal{U}_{3 B}}{\partial z}
$$

Expanding on these relations, the final output is obtained (in the instantaneous Earth-pointing reference frame):

$$
\begin{aligned}
\boldsymbol{a}_{3 B} & =\left\{a_{x}, a_{y}, a_{z}\right\} \\
a_{x} & =-\mu\left(\frac{-1+x}{\left(1-2 x+r^{2}\right)^{\frac{3}{2}}}-\frac{3 x^{2}}{r^{5}}+\frac{1-x}{r^{3}}\right) \\
a_{y} & =-y\left(\frac{\mu}{\left(1-2 x+r^{2}\right)^{\frac{3}{2}}}-\frac{\mu\left(3 x+r^{2}\right)}{r^{5}}\right) \\
a_{z} & =-z\left(\frac{\mu}{\left(1-2 x+r^{2}\right)^{\frac{3}{2}}}-\frac{\mu\left(3 x+r^{2}\right)}{r^{5}}\right)
\end{aligned}
$$

\subsection{Final Framework}

The accelerations used in the GVE-3B (Eqs. (4)) have to be written in the LVLH frame. However, $\boldsymbol{a}_{3 B}$ is described in the Earth-pointing reference frame, while the depiction of $\boldsymbol{a}_{L T}$ depends on the setting of the problem. For the sake of reaching a formula for $\boldsymbol{a}_{G V E}, \boldsymbol{a}_{L T}$ is here described in a barycentric, inertial Cartesian frame. Thus, some transformations have to be taken into account. 
For this, several rotation matrices are derived: $\mathbf{R}_{I}^{\oplus}$ is the rotation matrix from the inertial Cartesian $\left(O_{I x y z}\right)$ to the Earth-pointing frame $\left(O_{\oplus x y z}\right) ; \mathbf{R}_{e p h}^{I}$ is used to convert from the orbital plane $\left(O_{e p h}\right)$ to the inertial Cartesian

frame; $\mathbf{R}_{r \theta h}^{e p h}$ rotates a vector from the LVLH frame $\left(O_{r \theta h}\right)$ to the orbital plane one. These matrices are presented in Appendix B. Eqs. (19) present the transformations to the acceleration vectors by each rotation matrix.

$$
\boldsymbol{a}_{\oplus x y z}=\mathbf{R}_{I}^{\oplus} \boldsymbol{a}_{I}, \quad \boldsymbol{a}_{I x y z}=\mathbf{R}_{e p h}^{I} \boldsymbol{a}_{e p h}, \quad \boldsymbol{a}_{e p h}=\mathbf{R}_{r \theta h}^{e p h} \boldsymbol{a}_{r \theta h}
$$

The accelerations to use in Eqs. (4) in the LVLH frame are, thus, computed as follows:

$$
\boldsymbol{a}_{G V E}=\left(\mathbf{R}_{e p h}^{I} \mathbf{R}_{r \theta h}^{e p h}\right)^{-1}\left(\mathbf{R}_{I}^{\oplus^{-1}} \boldsymbol{a}_{3 B}+\boldsymbol{a}_{L T}\right)
$$

The state propagation is done in orbital elements. For a better understanding of the transformations involved and the overall framework, the flowchart of Figure 2 can be analysed.

\subsection{State Transition Matrix}

To correctly and efficiently adjust a trajectory in a desired motion model using, for instance, a differential correction method, the computation of the state transition matrix (STM) is necessary. The STM provides a linear mapping from time $t_{0}$ to a time $t$, establishing a relationship between initial and final deviations that can be used to adjust trajectories in order to match a final outcome. The propagation of the STM is described by the following equation:

$$
\dot{\boldsymbol{\Phi}}\left(t, t_{0}\right)=\mathbf{D} f(s(t)) \boldsymbol{\Phi}\left(t, t_{0}\right)
$$

where $\boldsymbol{s}$ is the state $\{x, y, z, \dot{x}, \dot{y}, \dot{z}\}, \mathbf{\Phi}\left(t, t_{0}\right)$ denotes the STM and $\mathbf{D} f(\boldsymbol{s}(t))$ is the Jacobian of the STM.

For the GVE-3B framework, the Jacobian of the STM $\left(\mathbf{D} f(\boldsymbol{s})_{3 B}\right)$ is presented in Appendix A of this work.

\subsection{Accuracy of the Model}

The GVE-3B equations are formulated as a low-fidelity tool for the computation of third-body perturbations in a system with small gravitational parameter. It is then important to figure out their performance as a function 


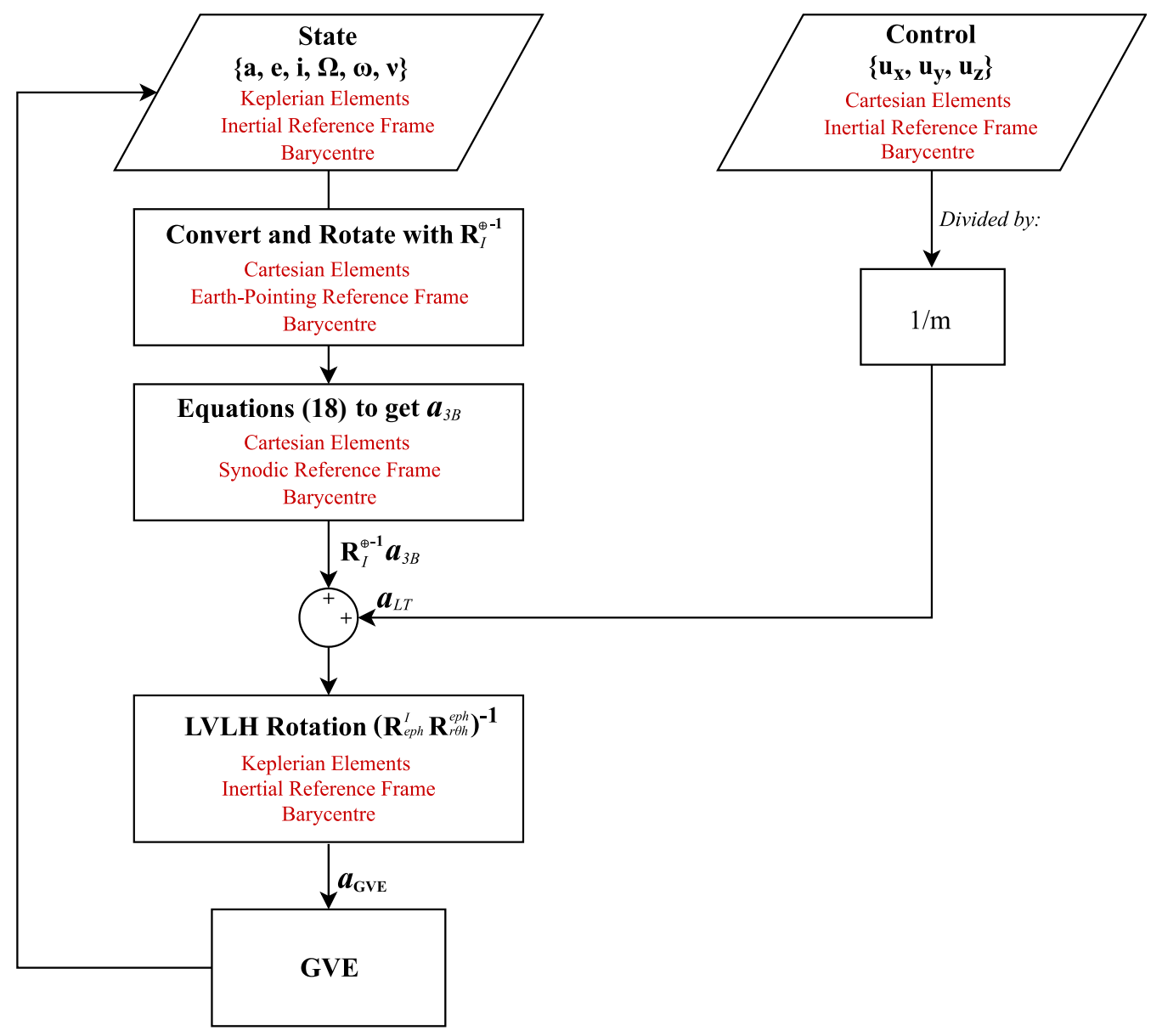

Figure 2: State propagation using the GVE Framework

of the distance to the perturbing body. As such, in the following analysis, the modelled motion will have no thrusting acceleration: the influence of the latter will be discussed in the asteroid mission trajectories of the following sections.

The GVE-3B error is contrasted to the 2BP error - these are defined as the distance between the position computed with the named model and with the CR3BP. This work claims that the accuracy of the 2BP is lacking in the vicinity of the Earth and up to very distant regions from its sphere of influence (the so-called perturbation region), making it unsuitable for the proposed asteroid trajectory design and presenting the GVE-3B framework as a more adequate alternative. 


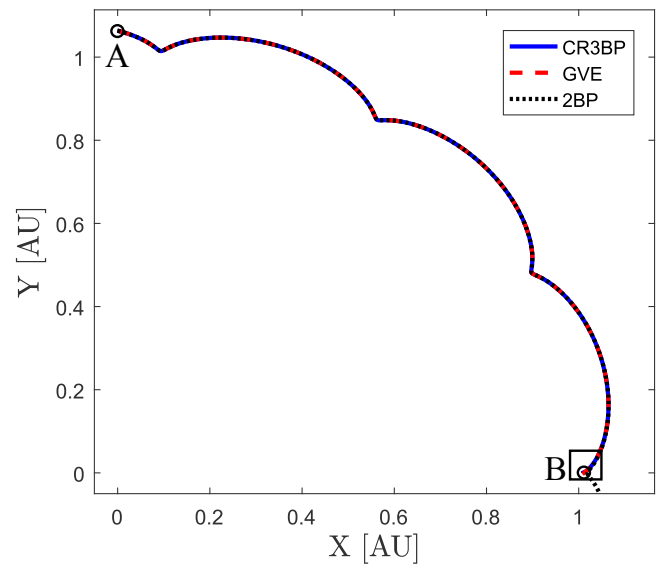

a) Full propagation from $\mathrm{A}$ to $\mathrm{B}$

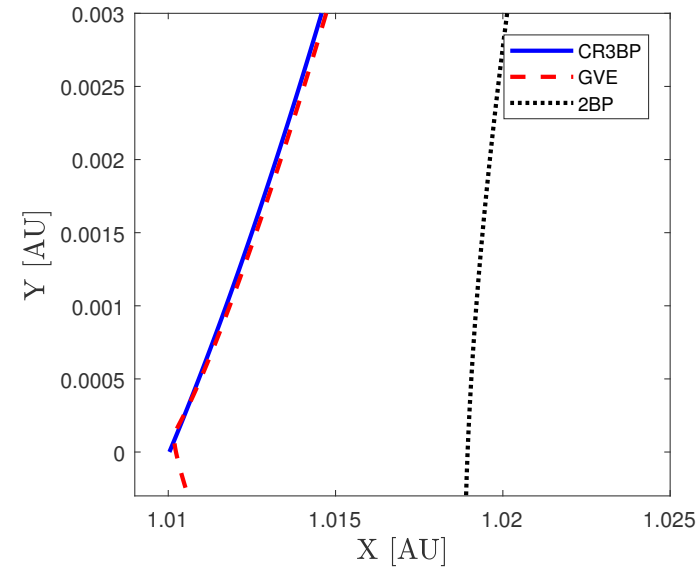

b) Zoom-in at point B

Figure 3: Propagation of the CR3BP, the GVE-3B and 2BP from point A to point B

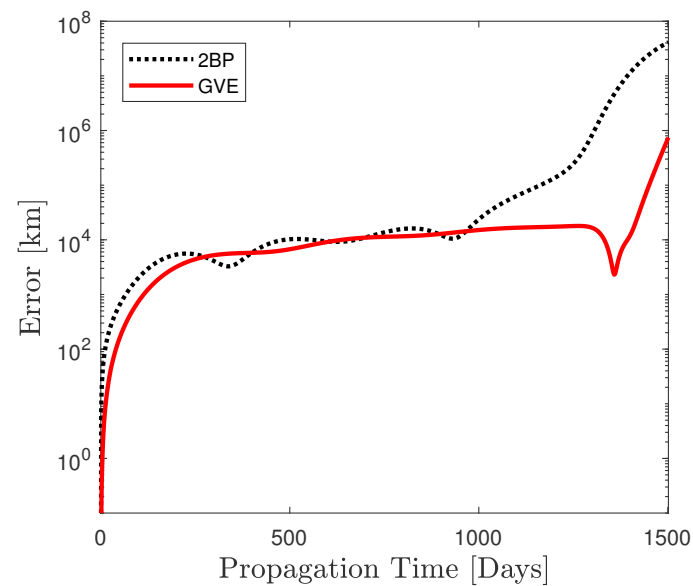

a) Error as a function of time

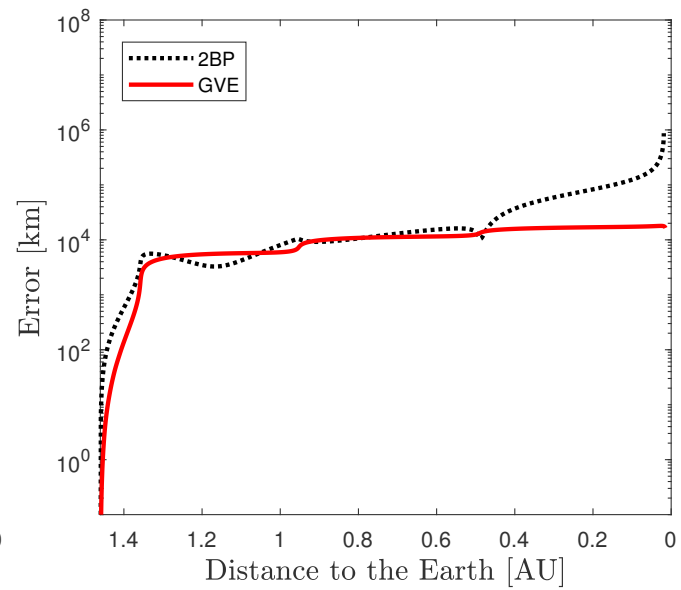

b) Error as a function of distance to the Earth, shown until the crossing of the X-axis

Figure 4: Propagation error for the 2BP and the GVE-3B for the trajectory in Figure 3

The first baseline trajectory for error comparison is found in Figure 3. The initial conditions are obtained in the following manner: the stable invariant manifold of the $L_{2}$ point is propagated backwards in time for a period of 1500 days, in the CR3BP - the final state is named point A. Then, from this point, the models were used to propagate forward, for the same time period. It follows that the end state should be the $L_{2}$ point (point B); however, when 
getting closer to the Earth, the difference between the GVE-3B and the 2BP increases, with the error of the latter shown to be especially large.

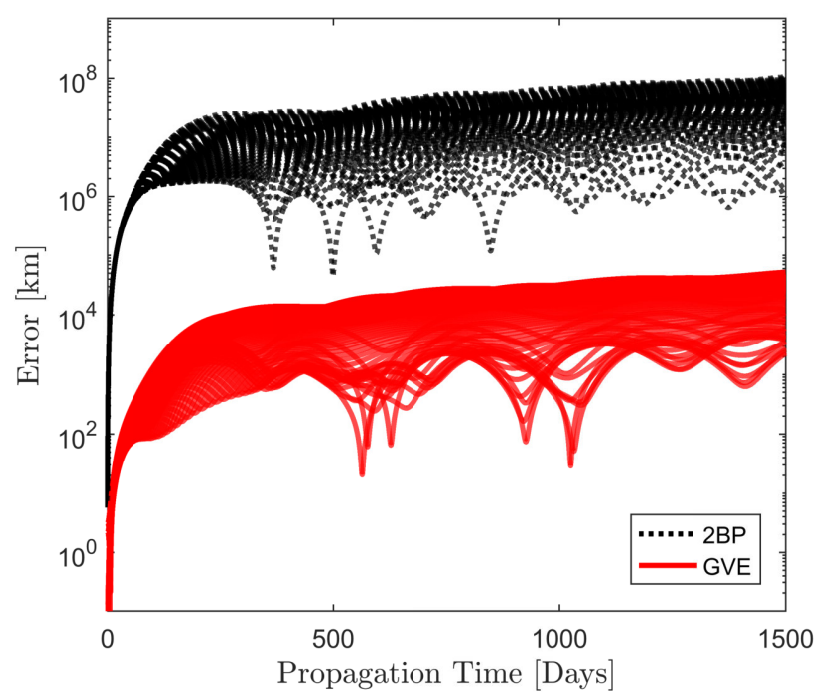

Figure 5: Propagation error (logarithmic scale) for the 2BP and the GVE-3B for 100 initial states in the Earth's vicinity

This error can be better analysed in Figure 4, where Figure 4 a) is plotted as a function of time and Figure $4 \mathrm{~b}$ ) as a function of the distance to the Earth. The GVE-3B error remains quite stable in the $10^{4} \mathrm{~km}$ value, until the $\mathrm{X}$-axis of the synodic reference frame is reached. As expected, when the trajectory approaches the Earth's sphere of influence ( $0.01 \mathrm{AU})$, there is an increase in the GVE-3B error. In contrast, the $2 \mathrm{BP}$ error increases faster, starting to do so much earlier.

In order to further establish the inadequacy of the $2 \mathrm{BP}$ in modelling trajectories close to the perturbing body, a different case study is performedthis time, starting at the vicinity of the Earth and ending away from it. For this purpose, a hundred initial conditions, each taken from a different point on the surface of the Earth's sphere of influence and never crossing it during the propagation time of 1500 days, are propagated without any initial velocity. The corresponding error for each trajectory can be seen in Figure 5. The $2 \mathrm{BP}$ error is much higher overall, since the trajectories start close to the disturbing body and consequently accumulate errors very quickly. In contrast, the GVE-3B error remains similar to the previously shown case, 
demonstrating its consistency in different application scenarios.

From these plots, it can be concluded that the CR3BP and GVE-3B model behave in a very similar way in the feasible region, which clearly evidences the quality of the framework presented in this paper and validates its use up to a very close region to the secondary. However, akin to the KM method (Alessi and Sánchez, 2015), the GVE-3B model is not adequate for use inside the sphere of influence of the Earth or for trajectories that remain for a long time in this vicinity.

\section{Trajectory Design for Asteroid Missions}

\subsection{Mission Summary}

The trajectory design for two asteroid missions is here presented: one for rendezvous, another for capture. Asteroid missions are invariably linked to the use of low-thrust propulsion systems, since the trajectory will benefit from their high exhaust velocity (Sánchez et al., 2018). Furthermore, both missions consider a spacecraft departing from (the rendezvous case) or arriving to (the capture case) one of the libration points of the Sun-Earth system. The closerange proximity operations that precede or conclude the missions are not considered in this work.

The asteroids were chosen using the Accessible NEAs NASA database ${ }^{3}$, which was searched to find objects whose estimated trajectory cost does not surpass $5 \mathrm{~km} \cdot \mathrm{s}^{-1}$. The choice fell on asteroids 2017 SV19 and 2018 AV2 as the rendezvous and capture targets. At the time of search, they were the most recently discovered ones with a clearly defined optical opportunity, i.e. a set calendar date in which the body will next be observable from the Earth.

Since both asteroids have semi-major axes greater than $1 \mathrm{AU}$, the $L_{2}$ point was selected as the departure for the rendezvous mission and the endgame for the capture one. This location is connected to several past missions (e.g. Herschel and Planck in 2009 (Pilbratt, 2001; Tauber, 2006)) and benefits from the existence of invariant manifold orbits that can be travelled without spending any fuel, both arriving to and departing from it.

\subsubsection{Asteroid Rendezvous}

Asteroid 2017 SV19 is an Amor asteroid discovered in September, 2017. Its diameter is in the 17-78 $\mathrm{m}$ range, with an estimated mass of 52,850 tonnes.

\footnotetext{
${ }^{3}$ cneos.jpl.nasa.gov/nhats, Accessed 01-09-2019
} 
The rendezvous trajectory starts with the spacecraft at the $L_{2}$ point, departing into an unstable invariant manifold orbit. At a certain time, the trajectory is changed to meet the nominal motion of the asteroid. The rendezvous happens during the asteroid's next close approach with the Earth, in 2040 .

\subsubsection{Asteroid Capture}

Asteroid 2018 AV2 is an Apollo asteroid discovered in January, 2018. Currently, there is little data regarding its composition and nature: it is known that its diameter is on the small range $(3.2-14 \mathrm{~m})$ and, assuming an average material composition, its mass is of about 318 tonnes (Bowell et al., 1989; Chesley et al., 2002). It is postulated that this object may likely be artificial (Jedicke et al., 2018); still, its dimensions make it a feasible notional body to study a capture mission, since it has to be small enough to be pushed by the used propulsion system.

The mission design can be described as the reverse of the rendezvous one: the spacecraft meets the asteroid at a certain point of the latter's nominal orbit and grabs it by any chosen means (Brophy et al., 2012; Cano et al., 2018). Then, the coupled system uses its propulsive capabilities to insert itself into a stable invariant manifold trajectory of the $L_{2}$ point, the target destination. This happens around the asteroid's next close approach with the Earth, starting in 2036.

Both bodies are moving in a very similar energy regime to the one of the Earth, making them great candidates to test the use of the GVE-3B framework. In order to better visualize and compare both mission scenarios, Figure 6 can be observed. The orbital transfer that will be designed in this chapter concerns the blue dashed segment from $t_{1}$ to $t_{2}$, while the rest of the motion uses no significant propulsion. The required steps in order to determine the initial and final points of the transfer (A and $\mathrm{B}$, respectively) will be explained in the following sections.

\subsection{Low-Thrust Trajectory Design}

The problem of designing a space transfer can be simply stated as the determination of a trajectory that satisfies some initial and final conditions, while minimizing a chosen parameter (Conway, 2010). This optimal control problem takes the information about the dynamical model to determine a set of controls that yield a trajectory obeying the initial conditions, constraints and bounds on the variables (Betts, 1998). This set of controls is generally 


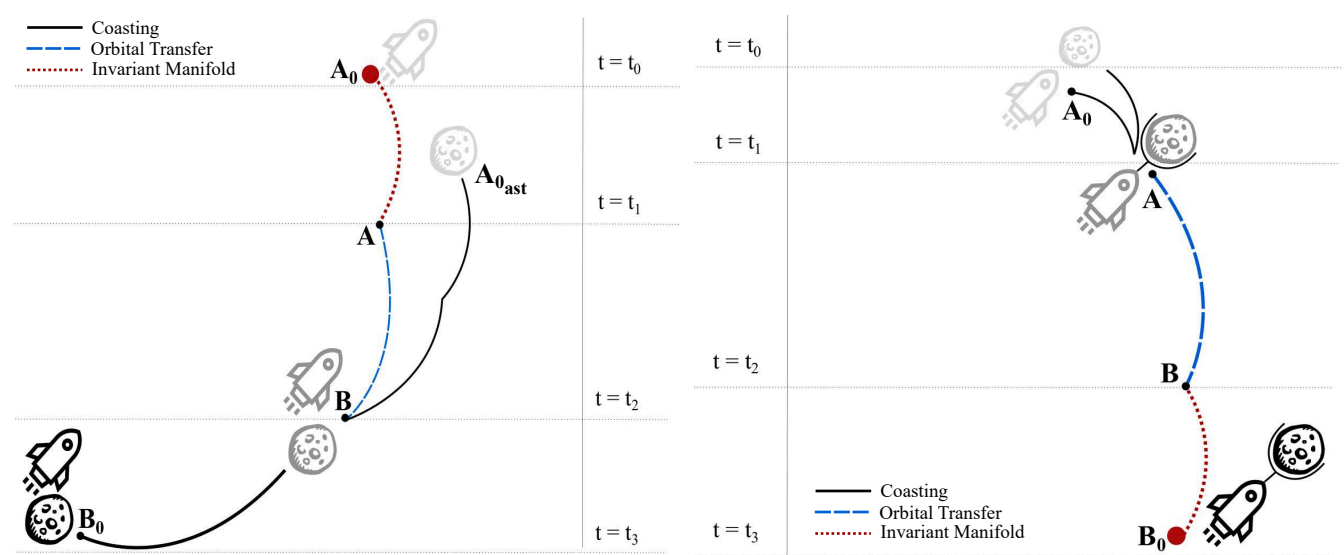

a) Rendezvous mission

b) Capture mission

Figure 6: Trajectory design for the suggested mission scenarios, showing the different segments and the times in which they happen

analogous to the spacecraft's acceleration, while the quantity to minimize relates to the overall fuel spent.

As previously stated, in order to design a low-thrust trajectory, feasible initial guesses that can be posteriorly transcribed and solved using an optimal control software solver are very useful. The computation of these first-guesses is here achieved by implementing the following sequential approach, detailed in the next sections:

Step 1: Transfer Optimisation. The optimal initial and final dates for the orbital transfer manoeuvre $\left(t_{1}\right.$ and $\left.t_{2}\right)$ are determined by computing several possible Lambert arcs with different boundary conditions and choosing the one with the lowest $\Delta v$.

Step 2: Sims-Flanagan-Inspired Approach. The set transfer dates are used to compute an initial guess trajectory akin to a Sims-Flanagan approach (Sims and Flanagan, 1999). This results in a transfer divided into equal-time segments, with an impulsive $\Delta v$ applied in each of them.

Step 3: Continuous Low-Thrust Transfer. The multiple-impulse transfer is transcribed to a continuous one, by converting the $\Delta v$ 's and segment times of the previous step into continuous accelerations and using a multiple shooting method. 


\subsubsection{Step 1: Transfer Optimisation}

Finding the best possible trajectory for both asteroid missions requires defining several potential starting and ending points and calculating the transfer costs between them. This means choosing the best Lambert arc out of different values of $t_{1}$ and $t_{2}$ and related asteroid ephemerides. In this case, the best arc is chosen as the one with the lowest $\Delta v$ : the corresponding values of $t_{1}$ and $t_{2}$ are the initial and final transfer times and the related asteroid ephemerides are fixed to the state of points A and B. These ephemerides remain the same throughout the following Steps.

More concretely, regarding the capture case: the starting point is to obtain the ephemerides of asteroid 2018 AV2 in a far-away position from the Earth, point $A_{0}$ in Figure 6 . This state is propagated forward in time to $t_{1}$ (point A). In a similar fashion, the invariant manifold of the $L_{2}$ point is propagated backwards to $t_{2}$ (point $B$ ). Then, the states corresponding to $t_{1}$ and $t_{2}$ are connected via a Lambert arc, obtaining the impulsive transfer cost. This is done for many different values of $t_{1}$ and $t_{2}$, yielding a porkchop plot of $\Delta v$ 's as a function of the initial and final dates.

An analogous methodology is implemented for the rendezvous case: the spacecraft's position is propagated forward from the $L_{2}$ point (at $t_{0}$ ) to $t_{1}$. Simultaneously, the ephemerides of asteroid 2017 SV19 are retrieved in a far-away position from the Earth $\left(\mathrm{B}_{0}\right)$ and propagated backwards to point B. The resulting ephemerides corresponding to $t_{1}$ and $t_{2}$ are connected with a Lambert arc.

The initial asteroid positions were retrieved from the Horizons JPL database $^{4}$. The porkchop plots were computed for the two models compared on this paper: the $2 \mathrm{BP}$ and the GVE-3B. For the former case, the propagations from $\mathrm{A}_{0}$ to $\mathrm{A}$ and $\mathrm{B}_{0}$ to $\mathrm{B}$ are done in the $2 \mathrm{BP}$, with the Lambert arc connecting $\mathrm{A}$ and $\mathrm{B}$ computed in the same model. For the latter case, the propagations from $\mathrm{A}_{0}$ to $\mathrm{A}$ and $\mathrm{B}_{0}$ to $\mathrm{B}$ are performed in the GVE-3B framework, and points $\mathrm{A}$ and $\mathrm{B}$ are connected with an arc in the same model. The latter is obtained by a single shooting method that employs the Jacobian of the STM in the GVE-3B model, as derived in Appendix A (Neves, 2019).

\footnotetext{
${ }^{4}$ https://ssd.jpl.nasa.gov/?horizons, Accessed 01-18-2019
} 


\subsubsection{Step 2: Sims-Flanagan-Inspired Approach}

The Sims-Flanagan method has been extensively used to further refine impulsive thrust first-guesses into low-thrust trajectories (Sims and Flanagan, 1999). This approach consists on adding several short impulses along the path, making it more similar to a low-thrust trajectory and, thus, more likely for an optimal control solver to converge easily (Hargraves and Paris, 1987). The trajectory is divided into segments with pre-defined durations. In the middle of each segment, a small impulsive manoeuvre is implemented. Each transfer is called a leg, defined from point A to B: the forward and backward propagations of these two locations meet at a match point, located usually halfway through the leg.

In other applications of the method, the problem is inserted into an optimiser with a constraint on the match point distance (Sims et al., 2006). In this work, instead of a match point, the forward and backward propagations from A and B are connected with a Lambert arc. Thus, the method is merely inspired by the Sims-Flanagan approach, and the named fundamental differences can be found on the schematic of Figure 7 .

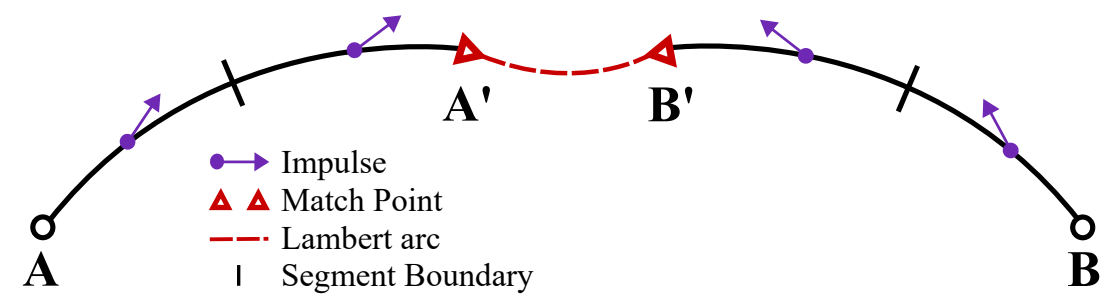

Figure 7: Sims-Flanagan-inspired trajectory scheme

For the mission design cases proposed in this paper, the Sims-Flanaganinspired trajectories are composed of 5 segments of equal duration (4 impulsive ones and the middle Lambert arc, as depicted in Figure 7). The application of this approach is further detailed in the following manner: first, points $\mathrm{A}$ and $\mathrm{B}$ are obtained from the optimal impulsive trajectory found via the porkchop plot. Then, a genetic algorithm ${ }^{5}$ is employed. The design variable is the vector of mid-segment impulses; the objective function propagates the motion forward from A and backwards from B using the GVE-3B model, implementing the $\Delta v$ 's in each segment. This yields match points A' and B',

\footnotetext{
${ }^{5}$ Using the MATLAB code suite (Chipperfield et al., 1994) with an initial population of 1000 parameters and the same number of maximum generations for convergence
} 
which are then connected via a Lambert arc in the GVE-3B model, obtained with a single shooting method. The goal is to minimize the sum of $\Delta v$ 's of the mid-segment impulses and the connecting arc (represented respectively by the purple arrows and the red dashed line of Figure 7).

\subsubsection{Step 3: Continuous Low-Thrust Transfer}

After obtaining the Sims-Flanagan-inspired solution, a low-thrust trajectory can finally be determined. Since the guiding motivation is to showcase the capabilities of the GVE-3B framework, the optimal control problem was simplified in a way that only the acceleration is optimised. The controlled trajectory design is computed in the following manner:

1. The impulses on each segment from the Sims-Flanagan-inspired approach are converted into continuous accelerations using the formula $\boldsymbol{a}=\frac{\boldsymbol{v}_{1}-\boldsymbol{v}_{0}}{\Delta t}$, in which $\boldsymbol{v}_{0}$ is the velocity at the beginning of the impulse, $\boldsymbol{v}_{1}$ is the velocity at the end of the impulse and $\Delta t$ is the segment's duration.

2. A is propagated forwards and B backwards, using the respective accelerations for each segment. The final points of the trajectory will be the new match points A" and B", different from the previous A' and B' since they are achieved with continuous accelerations.

3. A" and B" are linked using a multiple shooting method using the GVE3B model, which changes the thrusting accelerations to match the initial and final states, achieving a fully connected trajectory.

Multiple Shooting Method: As stated in Section 3.4, differential correction is necessary for various trajectory design applications. A multiple shooting method is a differential correction technique utilised to connect the initial and final points of a trajectory with multiple segments, by using several single shooting methods between patch points. In this way, the errors associated with the long integration of segments are reduced. Plus, the shape of the trajectory can be more easily manipulated by adding constraints to the different patch points. The overall schematic of this method can be found on Figure 8, from an initial disconnected guess to the fully linked trajectory.

In a multiple shooting scheme, the integration times can be fixed or variable. For the sake of simplicity, this paper utilises the first case. In this scenario, the vector of free variables corresponds to the linkage of each patch 


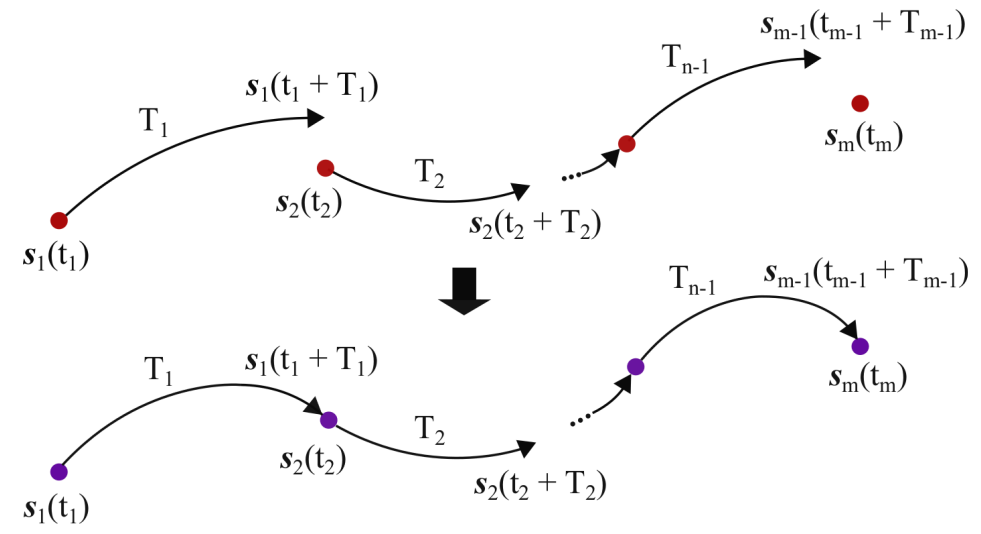

Figure 8: Multiple shooting scheme

point's state vector $\left(\boldsymbol{s}_{j}, j=1, \ldots, m\right)$. It becomes a column vector of $9 m$ rows when the position, velocity and acceleration are considered (where $m$ is the number of patch points).

$$
\boldsymbol{X}=\left[\begin{array}{lll}
\boldsymbol{s}_{1} & \ldots & \boldsymbol{s}_{m}
\end{array}\right]^{T}
$$

The solution to the multiple shooting scheme involves iterating through Equation 23 until the error $\left(\left\|\mathbf{G}\left(\boldsymbol{X}^{i+1}\right)\right\|\right)$ is smaller than a convergence tolerance $(\epsilon)$-in this paper, chosen as $\epsilon=10^{-8}$ :

$$
\boldsymbol{X}^{i+1}=\boldsymbol{X}^{i}-\mathrm{DG}\left(\boldsymbol{X}^{i}\right)^{-1} \mathbf{G}\left(\boldsymbol{X}^{i}\right)
$$

in which the superscript ${ }^{i}$ represents the iteration number, $\mathbf{G}(\boldsymbol{X})$ is the constraint vector and $\mathbf{D G}(\boldsymbol{X})$ is the Jacobian of the constraint vector.

Besides position and velocity continuity at the patch points, any other constraints can be added. The constraint vector takes the particular form of Eq. (24), where the first 3 conditions represent the patch point continuity requirements and the latter 2 establish that A" and B" are fixed. The number of patch points was chosen to be 3 , considering the trade-off between having a solution with more nodes or an easier convergence.

$$
\mathbf{G}(\boldsymbol{X})=\left[\begin{array}{c}
\boldsymbol{s}_{1}\left(t_{1}+T_{1}\right)-\boldsymbol{s}_{2}\left(t_{2}\right) \\
\boldsymbol{s}_{2}\left(t_{2}+T_{2}\right)-\boldsymbol{s}_{3}\left(t_{3}\right) \\
\boldsymbol{s}_{3}\left(t_{3}+T_{3}\right)-\boldsymbol{s}_{4}\left(t_{4}\right) \\
\boldsymbol{s}_{1}\left(t_{1}\right)-\boldsymbol{A}^{\prime \prime} \\
\boldsymbol{s}_{4}\left(t_{4}\right)-\boldsymbol{B}^{\prime \prime}
\end{array}\right]
$$


It is important to note that the accelerations are kept free for all the patch points. Thus, the Jacobian matrix of this constraint vector is presented in Eq. (25), where $\boldsymbol{\Phi}$ represents the STM of the GVE-3B framework computed in Section 3.4.

$$
\begin{aligned}
\mathbf{D G}(\boldsymbol{X}) & =\left[\begin{array}{cccccc}
\boldsymbol{\Phi}_{1,6 \times 9} & -\mathbf{I O}_{6 \times 9} & \mathbf{0}_{6 \times 9} & \mathbf{0}_{6 \times 9} \\
\mathbf{0}_{6 \times 9} & \boldsymbol{\Phi}_{2,6 \times 9} & -\mathbf{I O}_{6 \times 9} & \mathbf{0}_{6 \times 9} \\
\mathbf{0}_{6 \times 9} & \mathbf{0}_{6 \times 9} & \boldsymbol{\Phi}_{3,6 \times 9} & -\mathbf{I O}_{6 \times 9} \\
\mathbf{I O}_{6 \times 9} & \mathbf{0}_{6 \times 9} & \mathbf{0}_{6 \times 9} & \mathbf{0}_{6 \times 9} \\
\mathbf{0}_{6 \times 9} & \mathbf{0}_{6 \times 9} & & \mathbf{0}_{6 \times 9} & \mathbf{I O}_{6 \times 9}
\end{array}\right] \\
\mathbf{I O} & =\left[\begin{array}{ccccccccc}
1 & 0 & 0 & 0 & 0 & 0 & 0 & 0 & 0 \\
0 & 1 & 0 & 0 & 0 & 0 & 0 & 0 & 0 \\
0 & 0 & 1 & 0 & 0 & 0 & 0 & 0 & 0 \\
0 & 0 & 0 & 1 & 0 & 0 & 0 & 0 & 0 \\
0 & 0 & 0 & 0 & 1 & 0 & 0 & 0 & 0 \\
0 & 0 & 0 & 0 & 0 & 1 & 0 & 0 & 0
\end{array}\right]
\end{aligned}
$$

\section{Results and Discussion}

This section presents the results from each phase of the trajectory design approach for the asteroid rendezvous and capture. The benefits of using the GVE-3B framework as the model of motion for low-thrust design are highlighted, and fully optimised transfers are presented for asteroids 2017 SV19 and 2018 AV2.

Porkchop Plots: The first step in the trajectory design was to search for the best possible transfer dates for capture and rendezvous. These start at a named point $\mathrm{A}$ and end at point $\mathrm{B}$, following the coasting motion from points $\mathrm{A}_{0}$ and $\mathrm{B}_{0}$. As detailed in Section 4.2.1, the ephemerides of $\mathrm{A}$ and $\mathrm{B}$ are obtained from the propagation of these specific initial conditions; the transfer cost is yielded by an arc connecting A to B. For comparison purposes, the same propagations were done using the $2 \mathrm{BP}$ and the GVE-3B models.

The ephemerides of asteroids 2017 SV19 and 2018 AV2 were taken for the dates: 9 October, 2040 (corresponding to position $A_{0_{a s t}}$ of Figure 6 a)) and 6 April, 2036 (position $A_{0}$ of Figure 6 b)).

In order to generate the departure and arrival dates for the porkchops, the characteristics of each mission had to be considered. For the rendezvous case, the departure time coincides with leaving the manifold orbit, while the arrival date corresponds to the matching with the asteroid's motion. The reverse happens for the capture case. Given this, from their initial collection 


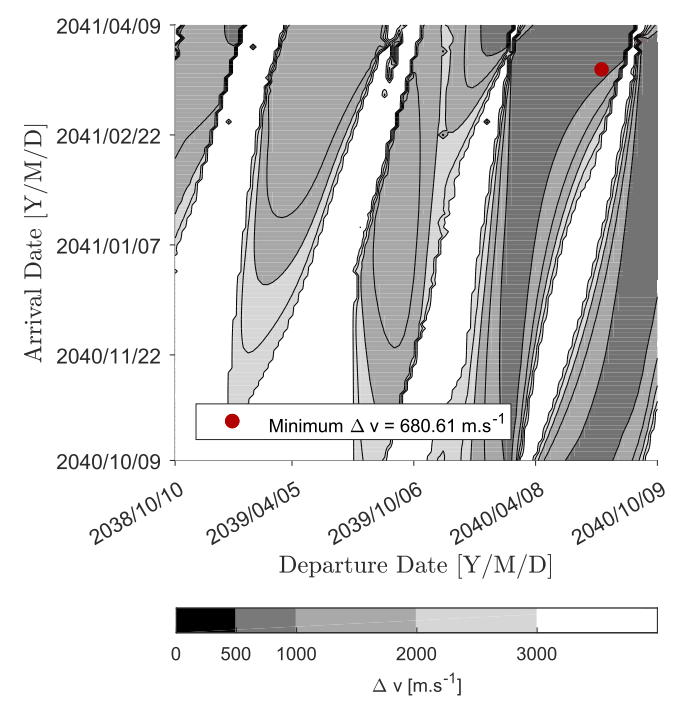

a) Rendezvous in the GVE-3B model

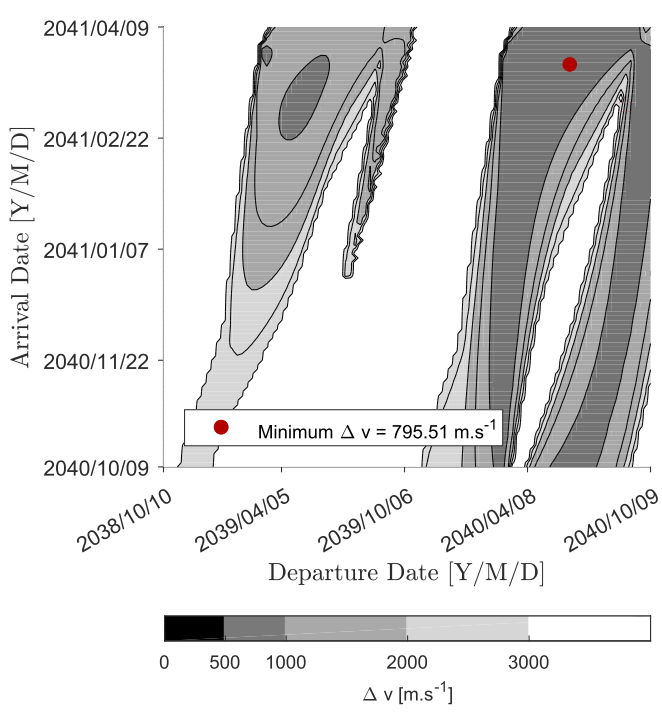

c) Rendezvous in the $2 \mathrm{BP}$ model

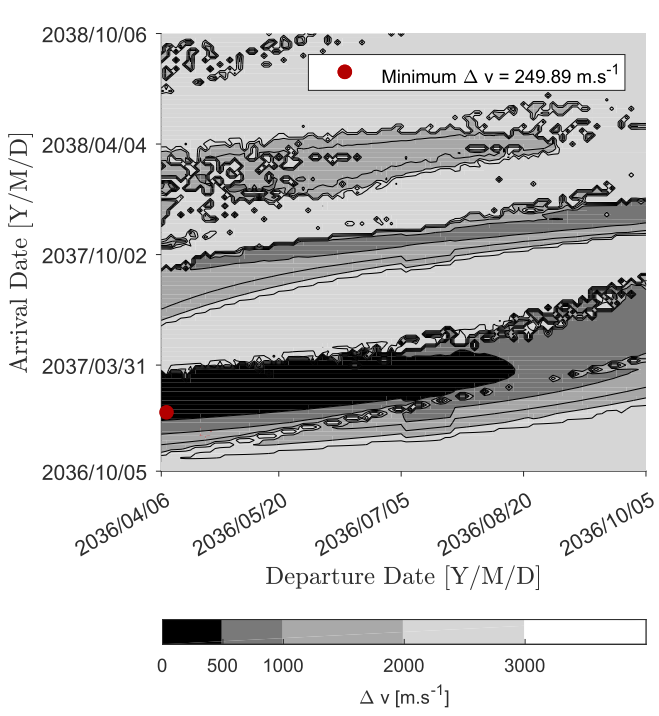

b) Capture in the GVE-3B model

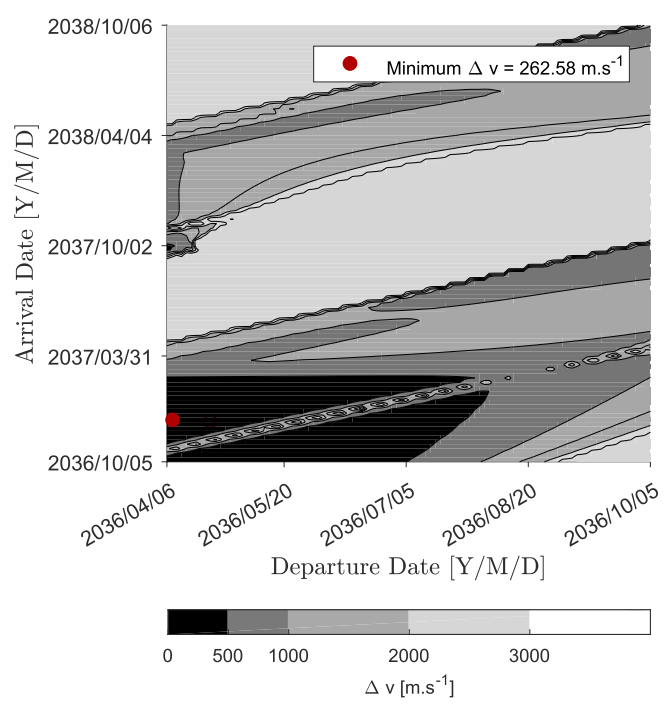

d) Capture in the 2BP model

Figure 9: Contour plots of the $\Delta v$ cost as a function of departure and arrival transfer dates (Year/Month/Day)

date, the ephemerides of each asteroid were propagated for a half-year period (corresponding to the arrival and departure time intervals of the rendezvous and capture missions, respectively). The manifold's state was sampled for a 
Table 1: Detailed minimal $\Delta v$ solutions for the porkchop plots computed in the GVE-3B model

\begin{tabular}{cc}
\hline Trajectory & Rendezvous \\
\hline Departure Date & $2040 / 09 / 21$ \\
Arrival Date & $2041 / 03 / 28$ \\
Departure State* & {$[1.55 \mathrm{E} 8,-4.57 \mathrm{E} 7,-202.59,7.74,27.10,-3.24 \mathrm{E}-6]$} \\
Arrival State* & {$[-1.40 \mathrm{E} 8,6.17 \mathrm{E} 7,2.65 \mathrm{E} 5,-12.19,-27.38,-0.68]$} \\
$\Delta v\left[\mathrm{~m} . \mathrm{s}^{-1}\right]$ & 680.61 \\
\hline Trajectory & Capture \\
\hline Departure Date & $2036 / 04 / 06$ \\
Arrival Date & $2037 / 01 / 19$ \\
Departure State* & {$[-1.18 \mathrm{E} 8,-1.01 \mathrm{E} 8,-3.05 \mathrm{E} 5,18.32,-22.65,-0.04]$} \\
Arrival State* & {$[-7.92 \mathrm{E} 7,1.30 \mathrm{E} 8,75.18,-25.35,-15.94,4.54 \mathrm{E}-5]$} \\
$\Delta v\left[{\left.\mathrm{~m} . \mathrm{s}^{-1}\right]}^{-1}\right]$ & 249.89 \\
\hline
\end{tabular}

* Sun-centred inertial reference frame, $\left[\mathrm{km}, \mathrm{km} \cdot \mathrm{s}^{-1}\right]$

1-year period. The arrival dates were taken to be at most 2 years after the latest departure date. In total, 10,000 Lambert arcs were computed, with 100 departure and 100 arrival points.

The contour plots indicating the transfer $\Delta v$ cost as a function of the initial and final manoeuvre dates can be found on Figure 9: images a) and b) depict the rendezvous and capture cases in which the arc connecting $\mathrm{A}$ to $\mathrm{B}$ is computed in the GVE-3B framework, while c) and d) show the computation using the 2BP. The Lambert arc optimisation allowed up to one revolution.

The optimal $\Delta v$ manoeuvres are marked with a red circle, and the details of the corresponding trajectories for the GVE-3B case can be found in Table 1 . The rendezvous trajectory yields a cost of about $681 \mathrm{~m} \cdot \mathrm{s}^{-1}$, while the capture requires $250 \mathrm{~m} \cdot \mathrm{s}^{-1}$. The optimal values obtained with the $2 \mathrm{BP}$ propagation are slightly different, as well as the optimal dates.

Preliminary Transfer Design: The ephemerides of points A and B were obtained for both mission scenarios, corresponding to the points detailed in Table 1. The same set of dates is used for the trajectories computed in the $2 \mathrm{BP}$, for an easier comparison. For each of these solutions, the SimsFlanagan-inspired approach was implemented.

In the GVE-3B model, the total $\Delta v$ for the optimal solutions was 865 


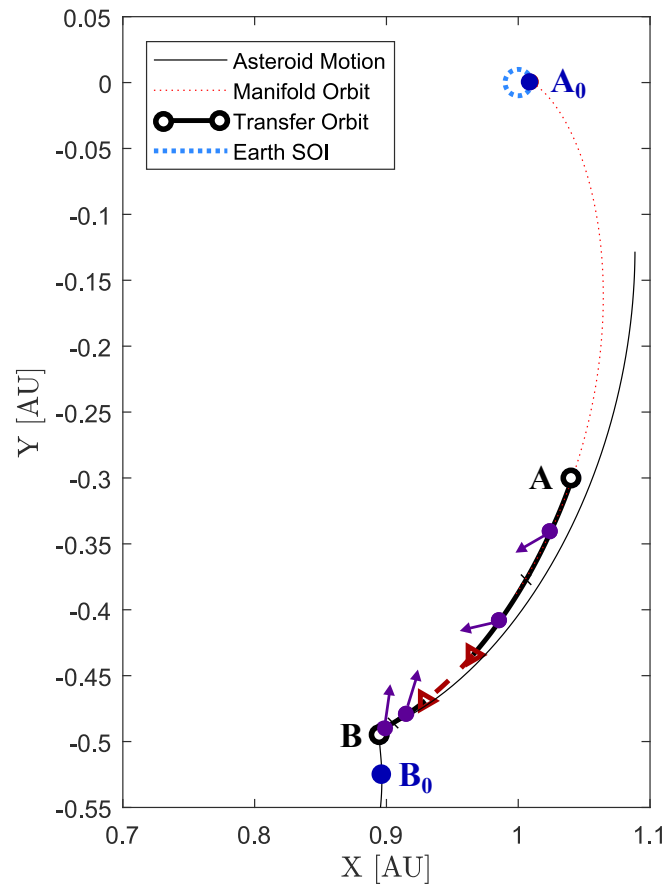

a) Rendezvous with Asteroid 2017 SV19

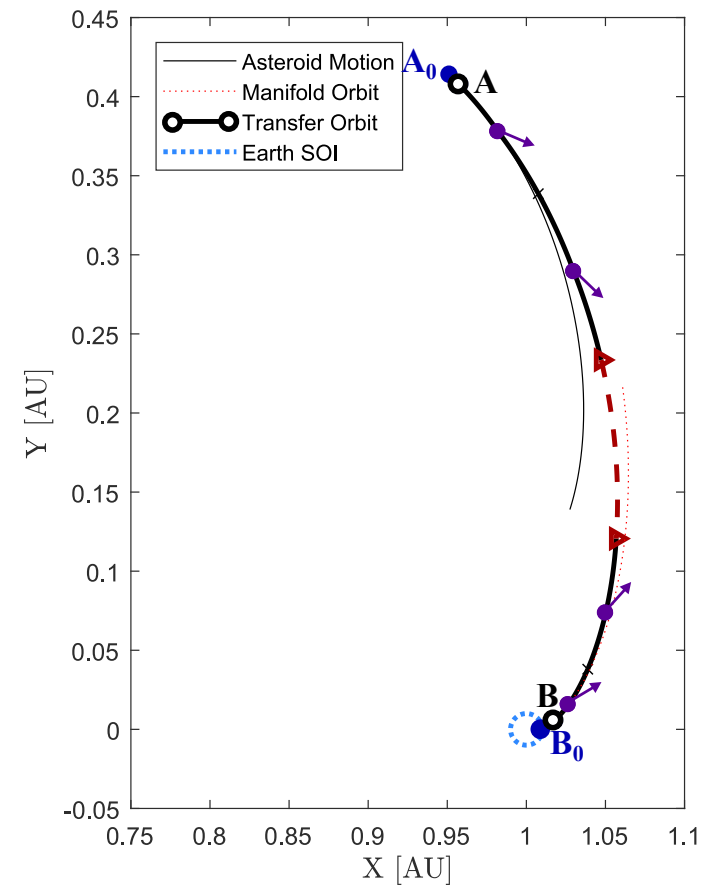

b) Capture of Asteroid 2018 AV2

Figure 10: Trajectory segments in the Sims-Flanagan-inspired approach

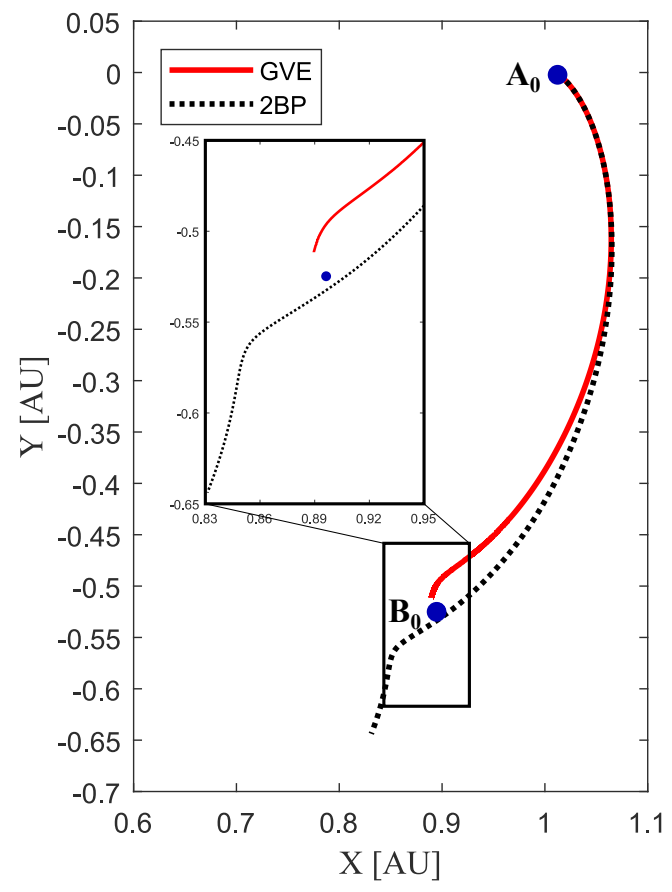

a) Rendezvous with Asteroid 2017 SV19

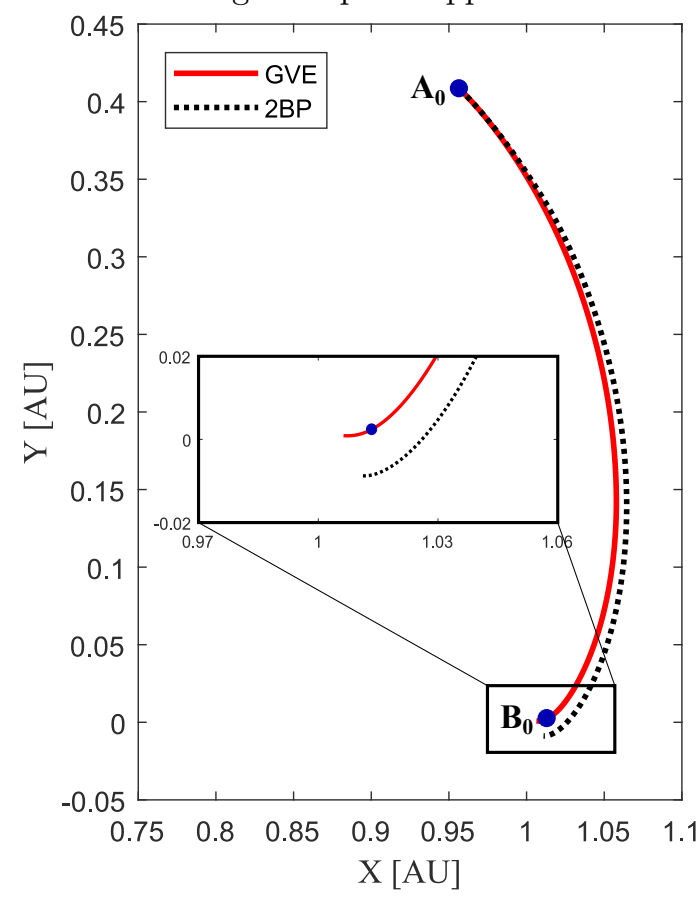

b) Capture of Asteroid 2018 AV2

Figure 11: Final trajectories with control laws determined with the GVE-3B framework (in red) and with the 2BP (in black), propagated with the CR3BP 
$\mathrm{m} \cdot \mathrm{s}^{-1}$ and $403 \mathrm{~m} \cdot \mathrm{s}^{-1}$, respectively for the rendezvous and capture cases. The obtained trajectories can be found in Figure 10. Figure 10 a) contains the rendezvous trajectory, from point $A_{0}$ in the invariant manifold orbit to point $\mathrm{B}_{0}$ of the nominal motion of 2017 SV19. Figure $10 \mathrm{~b}$ ) depicts the capture transfer starting from point $\mathrm{A}_{0}$ in the nominal motion of 2018 AV2 and finishing at point $\mathrm{B}_{0}$ of the invariant manifold orbit. The purple arrows indicate the small manoeuvres of the Sims-Flanagan-inspired approach, with the red dashed line outlining the Lambert arc that connects patch points A' and B'.

As detailed in the Step 3 of Section 4.2, two control laws for the transfer part of the motion (A to B) were devised, using the Sims-Flanagan-inspired approach and the multiple shooting method - again, one uses the GVE-3B framework, while the other employs the $2 \mathrm{BP}$.

Figure 11 shows the propagation of both control sequences with the baseline CR3BP model. The trajectories that employ the GVE-3B control law end up much closer to the target than the corresponding 2BP ones, something particularly evident in the asteroid rendezvous case.

Optimal Control Solver: To study the convergence of the optimal control problem, a preliminary analysis employing the optimal control solver GPOPSII was performed.

The optimal control solver uses the previously obtained trajectories from A to B as a first-guess to achieve the desired motion in the CR3BP model. The inputs of the solver are these trajectory states, the control history vectors and the ephemerides of points A and B.

In practical terms, both the state and acceleration vectors are first discretized into 200 points each. These are then used by the GPOPS-II optimizer; in this implementation, the objective was only to have the state vector converge to target point $\mathrm{B}$ within a $1 \mathrm{~km}$ error radius; no minimization of acceleration was considered and no other constraints were imposed, such as maximum thrust or spacecraft's mass. This choice intended to make the convergence faster and highlight the importance of the first-guess inputs.

Figure 12 details the first-guess control histories and the GPOPS-II solution for each of the previously determined control laws (computed in the GVE-3B and 2BP). When compared to the first-guesses, the trajectory segments can be easily distinguished. In the cases of Figures 12 b), c) and d), where the multiple shooting phase leads to acceleration peaks, the solver returns a solution that smooths them out. 

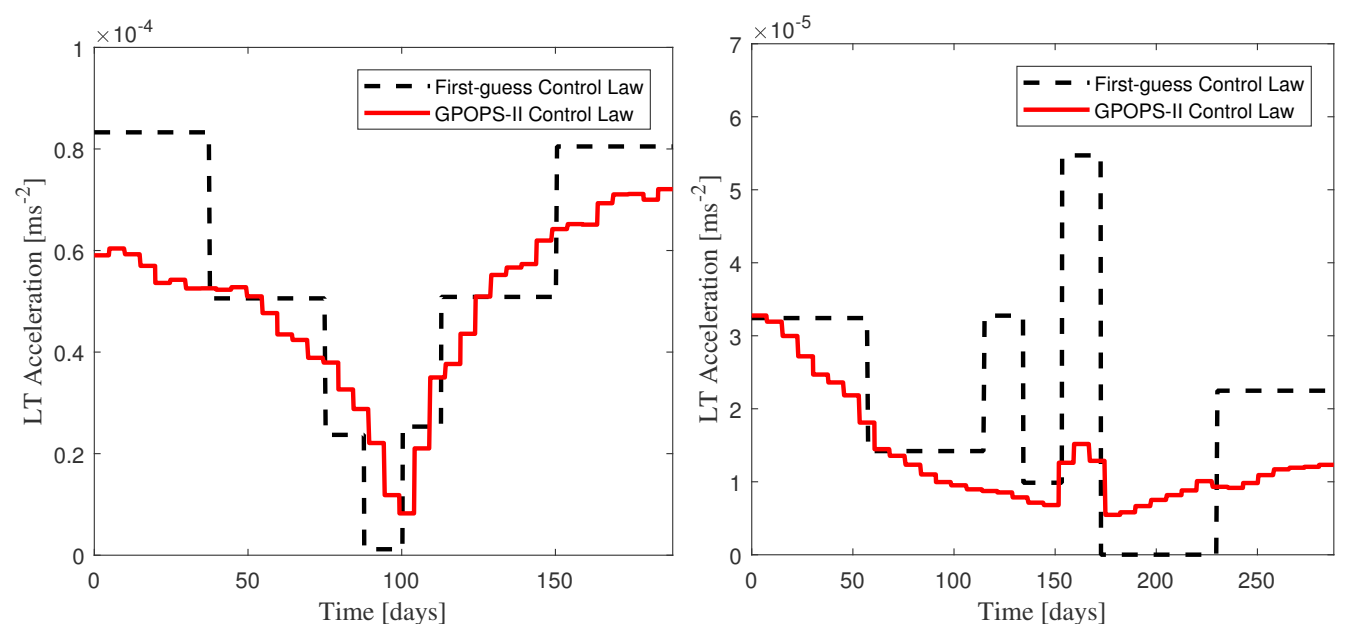

a) Control law for rendezvous in the GVE-3B model

b) Control law for capture in the GVE-3B model

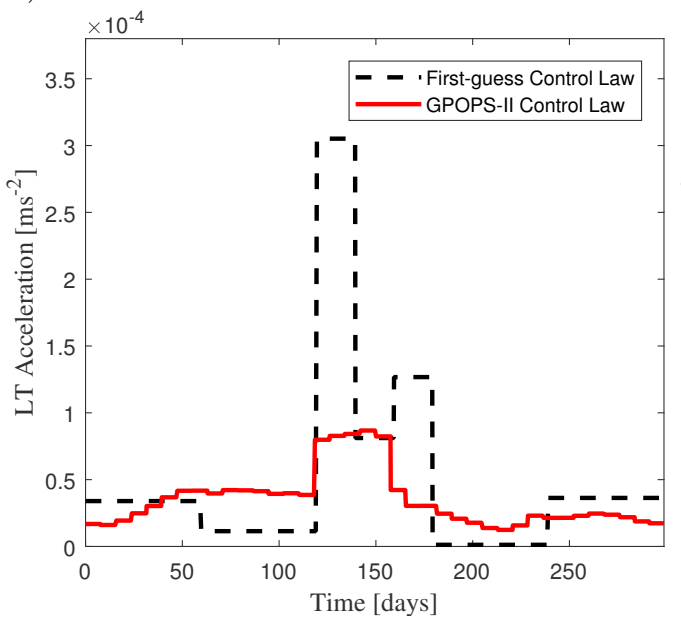

c) Control law for rendezvous in the $2 \mathrm{BP}$ model

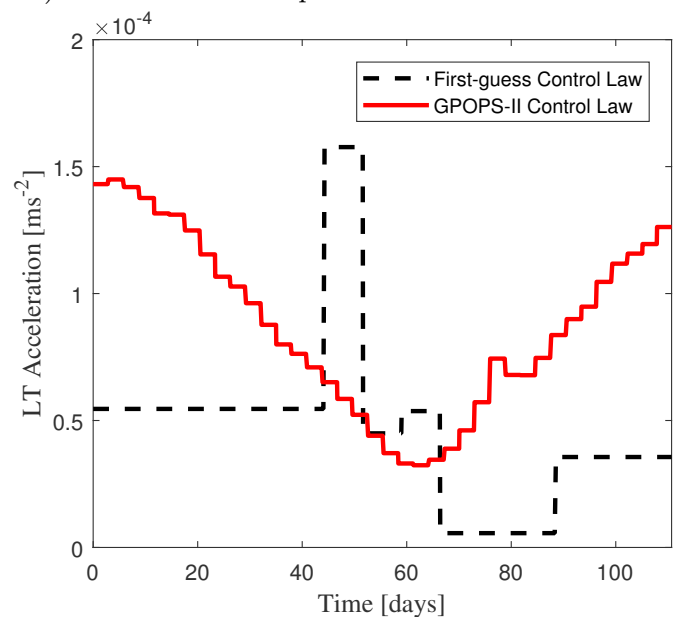

d) Control law for capture in the 2BP model

Figure 12: Acceleration norm throughout the transfer from A to B

Final Considerations: The $\Delta v$ solutions found at each of the Steps in this preliminary mission design are summarized in Table 2.

The optimal control solver converges easily for the GVE-3B and 2BP control laws, with a similar number of iterations - however, the solution of the former model is more optimal in terms of total acceleration. In the capture case, the final GVE-3B control law yields a $\Delta v$ of only $362 \mathrm{~m} \cdot \mathrm{s}^{-1}$, compared to the $884 \mathrm{~m} . \mathrm{s}^{-1}$ of the $2 \mathrm{BP}$ solution. The difference is not as pronounced in the rendezvous case, since the performance improvement generated by employing 
Table 2: $\Delta v$ solutions for each of the steps in the trajectory design, from the porkchop computation (Step 1) to the optimal control solver (GPOPS-II).

\begin{tabular}{cccccc}
\hline \multicolumn{2}{c}{$\Delta v^{*}$} & Step 1 & Step 2 & Step 3 & GPOPS-II \\
\hline \multirow{2}{*}{ Rendezvous } & GVE-3B model & 680.61 & 864.83 & 916.73 & 857.86 \\
& 2BP model & 680.62 & 906.85 & 1311.38 & 954.18 \\
\multirow{2}{*}{ Capture } & GVE-3B model & 249.89 & 403.41 & 505.03 & 361.68 \\
& 2BP model & 303.69 & 381.71 & 451.03 & 883.78 \\
\hline
\end{tabular}

*All of the trajectories start and finish at the A and B dates found to be the best solution of the GVE-3B porkchop plot, in Table 1

the GVE-3B model will depend on how close the trajectories move in regards to the Earth, and for how long they are propagated.

Furthermore, although no constrains were included for the hypothetical spacecraft used, the maximum acceleration showcased in the GVE-3B control law is adequate for a ship with similar specifications to that of BepiColombo ${ }^{6}$, pointing towards the feasibility of a possible mission even without further trajectory optimizations.

The trajectories presented intend to showcase the usage of the GVE3B framework for mission trajectory design as a valuable model to study motions under a third-body perturbation. Since the goal was to highlight the model of motion, the trajectory design was simplified in some respects. For instance, the setting up of optimal control solver parameters and cost function can be more detailed and actively try to minimize the total mission acceleration. Future implementations could see the first-guess improved by having the multiple shooter be used throughout the entire motion, so that a smoother control is attained. In addition to this, it is clear that fixing the departure and arrival dates of the first-guess and optimal control solvers is a very strict constraint that prevents finding even better solutions. Still, it is shown that the GVE-3B equations allow the generation of control laws that are substantially more accurate when propagated in high-fidelity dynamics.

\footnotetext{
${ }^{6}$ https://sci.esa.int/web/bepicolombo/-/47346-fact-sheet
} 


\section{Conclusions}

This paper presents a novel formulation of the third-body perturbation, ideal for a GVE approach to the propagation of trajectories within lowenergy regions and subject to low-thrust propulsive accelerations. This is especially useful for mission design in low-energy regimes, where the simultaneous perturbation of both primaries cannot be approximated by a lower fidelity method like the $2 \mathrm{BP}$.

The nature of the GVE equations makes them very advantageous for low-thrust trajectory design. Particularly, their formulation in Keplerian elements provides an intuitive observation of the trajectory evolution and an easy definition of boundaries and constraints, facilitating the set up and convergence of the optimal control problem solver. Thus, its utilisation for preliminary mission design can be extremely valuable, providing important insights into the time evolution of the orbital elements.

The developed equations of motion are valid for planetary systems with gravitational parameters of orders of magnitude similar to the Sun-Earth one. This makes them ideal for the computation of low-thrust trajectories for asteroid capture or rendezvous missions to the libration points, since the trajectory design can target low-energy invariant manifold structures very close to the periodic orbit.

Future work may consider the improvement of the preliminary trajectory design techniques presented in this paper. One idea would be to further minimize the mission $\Delta v$ costs by picking out optimal libration point orbits (LPOs) and connected manifold orbits (i.e. closer to the asteroids' energy), instead of the generic $L_{2}$ point. Another would be to free the times corresponding to each manoeuvre instead of having them fixed, which may be sub-optimal for the final solution, as low-thrust trajectories are generally slower than impulsive ones.

With respect to the GVE-3B framework, it is also envisaged to integrate it with multiple planetary systems to perform trajectory design for Jovian or Saturnian moon tours. Furthermore, given the existence of singularities for the inclination and longitude of the ascending node elements in the GVE equations, an application of modified orbital elements can be considered. 


\section{Appendix}

A - Jacobian of the State Transition Matrix for the GVE-3B model

The Jacobian of the STM for the GVE-3B model takes the form of Eq. (27). The size of $\mathbf{D} f(s(t))_{3 B}$ is $9 \mathrm{x} 9$ instead of the commonly found $6 \mathrm{x} 6$, since it includes the acceleration component on the state vector, as required by the multiple shooting scheme presented in this work.

$$
\mathbf{D} f(\boldsymbol{s}(t))_{3 B}=\left[\begin{array}{ccc}
\mathbf{0}_{3 \times 3} & \mathbf{I}_{3 \times 3} & \mathbf{0}_{3 \times 3} \\
\mathbf{R}_{I}^{\oplus^{-1}} \mathbf{A}_{3 B} & \mathbf{0}_{3 \times 3} & \mathbf{I}_{3 \times 3} \\
\mathbf{0}_{3 \times 3} & \mathbf{0}_{3 \times 3} & \mathbf{0}_{3 \times 3}
\end{array}\right], \mathbf{A}_{3 B}=\left[\begin{array}{lll}
\partial_{x} a_{x} & \partial_{y} a_{x} & \partial_{z} a_{x} \\
\partial_{x} a_{y} & \partial_{y} a_{y} & \partial_{z} a_{y} \\
\partial_{x} a_{z} & \partial_{y} a_{z} & \partial_{z} a_{z}
\end{array}\right]
$$

where $\partial_{\beta} a_{\alpha}(\alpha \in\{x, y, z\}$ and $\beta \in\{x, y, z\})$ are the first partial derivatives of the accelerations $\left\{a_{x}, a_{y}, a_{z}\right\}$ presented in Eq. (18). These are expanded into the following:

$$
\begin{aligned}
& \partial_{x} a_{x}=\mu\left(\frac{3(x-1)^{2}}{r_{3}^{5}}-\frac{1}{r_{3}^{3}}-\frac{15 x^{3}}{r^{7}}+\frac{9 x-3 x^{2}}{r^{5}}+\frac{1}{r^{3}}\right) \\
& \partial_{y} a_{x}=-3 y \mu\left(\frac{1-x}{r_{3}^{5}}+\frac{5 x^{2}}{r^{7}}-\frac{1-x}{r^{5}}\right) \\
& \partial_{z} a_{x}=-3 z \mu\left(\frac{1-x}{r_{3}^{5}}+\frac{5 x^{2}}{r^{7}}-\frac{1-x}{r^{5}}\right) \\
& \partial_{x} a_{y}=y \mu\left(\frac{3(x-1)}{r_{3}^{5}}+\frac{3+2 x}{r^{5}}-\frac{5 x\left(3 x+r^{2}\right)}{r^{7}}\right) \\
& \partial_{y} a_{y}=\mu\left(\frac{3 x+r^{2}}{r^{5}}-\frac{1}{r_{3}^{3}}+3 y^{2}\left(\frac{1}{r_{3}^{5}}-\frac{5 x+r^{2}}{r^{7}}\right)\right) \\
& \partial_{z} a_{y}=3 y z \mu\left(\frac{1}{r_{3}^{5}}-\frac{\left.5 x+r^{2}\right)}{r^{7}}\right) \\
& \partial_{x} a_{z}=z \mu\left(\frac{3(x-1)}{r_{3}^{5}}+\frac{3+2 x}{r^{5}}-\frac{5 x\left(3 x+r^{2}\right)}{r^{7}}\right) \\
& \partial_{y} a_{z}=3 y z \mu\left(\frac{1}{r_{3}^{5}}-\frac{\left.5 x+r^{2}\right)}{r^{7}}\right) \\
& \partial_{z} a_{z}=\mu\left(\frac{3 x+r^{2}}{r^{5}}-\frac{1}{r_{3}^{3}}+3 z^{2}\left(\frac{1}{r_{3}^{5}}-\frac{5 x+r^{2}}{r^{7}}\right)\right)
\end{aligned}
$$

where $r_{3}=\sqrt{1-2 x+x^{2}+y^{2}+z^{2}}$. 


\section{$B$ - Transformation between Coordinate Frames}

The rotation matrix from the inertial Cartesian frame to the Earthpointing frame is given by:

$$
\mathbf{R}_{I}^{\oplus}=\left[\begin{array}{ccc}
\cos \left(t+\nu_{\oplus_{0}}\right) & -\sin \left(t+\nu_{\oplus_{0}}\right) & 0 \\
\sin \left(t+\nu_{\oplus_{0}}\right) & \cos \left(t+\nu_{\oplus_{0}}\right) & 0 \\
0 & 0 & 1
\end{array}\right]
$$

The rotation of the LVLH frame to the orbital plane frame is done using Eq. (30). Eq. (31) converts vectors from the orbital plane to the inertial reference frame, complying with the definitions in Battin (Battin, 1999).

$$
\begin{gathered}
\mathbf{R}_{r \theta h}^{e p h}=\left[\begin{array}{ccc}
\cos \nu & -\sin \nu & 0 \\
\sin \nu & \cos \nu & 0 \\
0 & 0 & 1
\end{array}\right] \\
\mathbf{R}_{e p h}^{I}=\left[\begin{array}{ccc}
\cos \Omega & -\sin \Omega & 0 \\
\sin \Omega & \cos \Omega & 0 \\
0 & 0 & 1
\end{array}\right]\left[\begin{array}{ccc}
1 & 0 & 0 \\
0 & \cos i & -\sin i \\
0 & \sin i & \cos i
\end{array}\right]\left[\begin{array}{ccc}
\cos \omega & -\sin \omega & 0 \\
\sin \omega & \cos \omega & 0 \\
0 & 0 & 1
\end{array}\right]
\end{gathered}
$$

\section{References}

Alessi, E.M., Sánchez, J., 2015. Semi-Analytical Approach for Distant Encounters in the Spatial Circular Restricted Three-Body Problem. Journal of Guidance, Control, and Dynamics 39, 351-359. doi:10.2514/1. G001237.

Alvarez, L., Alvarez, W., Asaro, F., Michel, H., 1980. Extraterrestrial Cause for the Cretaceous-Tertiary Extinction. Science 208, 1095-1108. doi:10. 1126/science.208.4448.1095.

Battin, R.H., 1999. An Introduction to the Mathematics and Methods of Astrodynamics. AIAA, Virginia, USA.

Betts, J.T., 1998. Survey of Numerical Methods for Trajectory Optimization. Journal of Guidance, Control and Dynamics 21, 193-207. doi:10.2514/2. 4231.

Bowell, E., Hapke, B., Domingue, D., Lumme, K., Peltoniemi, J., Harris, A.W., 1989. Application of Photometric Models to Asteroids, in: Asteroids II, pp. 524-556. 
Brophy, J., Culick, F., Friedman, L., Allen, C., Baughman, D., Bellerose, J., et al., 2012. Asteroid Retrieval Feasibility Study. Report. Keck Institute for Space Studies, Califonia Institute of Technology, Jet Propulsion Laboratory.

Cano, J., Cunill, J., Diaz, A.J., Golemis, A., Gupta, S., Innes, D., Maiden, D., March, K., Rael, H., Shawe, J., Sierra, V., Torrents, A., Rossi, E.Z., Machuca, P., Neves, R., Sánchez, J.P., 2018. ARTEMIS: A Complete Mission Architecture to Bridge the Gap Between Humanity and NearEarth Asteroids, in: Proceedings of the $69^{\text {th }}$ International Astronautical Congress, Bremen, Germany.

Chesley, S.R., Chodas, P.W., Milani, A., Valsecchi, G.B., Yeomans, D.K., 2002. Quantifying the Risk posed by Potential Earth Impacts. Icarus 159, 423-432. doi:10.1006/icar.2002.6910.

Chipperfield, A., Fleming, P., Pohlheim, H., Fonseca, C., 1994. Genetic Algorithm TOOLBOX For Use with MATLAB .

Chirikov, B., Vecheslavov, V.V., 1993. Theory of Fast Arnold Diffusion in Many-Frequency Systems. Journal of Statistical Physics 71, 243-258. doi:10.1007/BF01048098.

Conway, B.A., 2010. Spacecraft Trajectory Optimization. Cambridge University Press, New York, USA. doi:10.1017/CB09780511778025.

Dachwald, B., 2004. Low-Thrust Trajectory Optimization and Interplanetary Mission Analysis Using Evolutionary Neurocontrol. Ph.D. thesis. Institut für Raumfahrttechnik, Universität der Bundeswehr, München.

Elvis, M., 2012. Let's Mine Asteroids-For Science and Profit. Nature 485, 549-550.

Gao, Y., 2007. Near-Optimal Very Low-Thrust Earth-Orbit Transfers and Guidance Schemes. Journal of Guidance, Control, and Dynamics 30, 529539. doi:doi.org/10.2514/1.24836.

Geffroy, S., Epenoy, R., 1997. Optimal Low-Thrust Transfers with Constraints - Generalization of Averaging Techniques. Acta Astronautica 41, 133-149. doi:doi .org/10.1016/S0094-5765(97)00208-7. 
Glassmeier, K.H., Boehnhardt, H., Koschny, D., Kührt, E., Richter, I., 2007. The Rosetta Mission: Flying Towards the Origin of the Solar System. Space Science Reviews 128, 1-21. doi:10.1007/s11214-006-9140-8.

Gomez, G., Koon, W.S., Lo, M.W., Marsden, J.E., Masdemont, J., Ross, S.D., 2001. Invariant Manifolds, the Spatial Three-Body Problem and Space Mission Design. 109, American Astronautical Society.

Hargraves, C.R., Paris, S.W., 1987. Direct Trajectory Optimization Using Nonlinear Programming and Collocation. Journal of Guidance, Control, and Dynamics 10, 338-342. doi:10.2514/3.20223.

Herman, A.L., Spencer, D.B., 2002. Optimal, Low-Thrust Earth-Orbit Transfers Using Higher-Order Collocation Methods. Journal of Guidance, Control, and Dynamics 25, 40-47. doi:doi .org/10.2514/2.4873.

Jedicke, R., Bolin, B.T., Bottke, W.F., Chyba, M., Fedorets, G., Granvik, M., Jones, L., Urrutxua, H., 2018. Earth's Minimoons: Opportunities for Science and Technology. Frontiers in Astronomy and Space Sciences 5, 13. doi:10.3389/fspas. 2018.00013.

Kawaguchi, J., Fujiwara, A., Uesugi, T., 2008. Hayabusa - Its Technology and Science Accomplishment Summary and Hayabusa-2. Acta Astronautica 62, 639-647. doi:10.1016/j.actaastro.2008.01.028.

Koon, W., Lo, M., Marsden, J., Ross, S.D., 2000. Heteroclinic Connections between Periodic Orbits and Resonance Transitions in Celestial Mechanics. Chaos: An Interdisciplinary Journal of Nonlinear Science 10, 427-469. doi:10.1063/1.166509.

Koon, W., Lo, M., Marsden, J., Ross, S.D., 2008. Dynamical Systems, the Three-Body Problem and Space Mission Design. Marsden Books doi:10 . 1142/9789812792617_0222.

Koon, W.S., Lo, M.W., Marsden, J.E., Ross, S.D., 2001. Low Energy Transfer to the Moon. Celestial Mechanics and Dynamical Astronomy 81, 63-73. doi:10.1023/A : 1013359120468.

Lewis, J., Hutson, M., 1993. Asteroidal Resource Opportunities Suggested by Meteorite Data. University of Arizona Press, Tucson Arizona. pp. 523 -542 . 
Nesvornỳ, D., Vokrouhlickỳ, D., Morbidelli, A., 2007. Capture of Irregular Satellites during Planetary Encounters. The Astronomical Journal 133, 1962. doi:10.1086/512850.

Neves, R., 2019. Multi-Fidelity Modelling of Low-Energy Trajectories for Space Mission Design. Ph.D. thesis. Cranfield University.

Neves, R., Sánchez, J.P., 2018. Multifidelity Design of Low-Thrust Resonant Captures for Near-Earth Asteroids. Journal of Guidance, Control, and Dynamics , 1-12doi:10.2514/1.G003599.

Noton, M., 2012. Spacecraft Navigation and Guidance. Springer Science \& Business Media. doi:10.1007/978-1-4471-1583-0.

Patterson, M., Rao, A., 2014. GPOPS-II: A MATLAB Software for Solving Multiple-Phase Optimal Control Problems using hp-Adaptive Gaussian Quadrature Collocation Methods and Sparse Nonlinear Programming. ACM Transactions on Mathematical Software (TOMS) doi:10. $1145 / 2558904$.

Petrosky, T.Y., Broucke, R., 1987. Area-Preserving Mappings and Deterministic Chaos for Nearly Parabolic Motions. Celestial Mechanics and Dynamical Astronomy 42, 53-79. doi:10.1007/BF01232948.

Pilbratt, G.L., 2001. The Herschel Mission, Scientific Objectives, and this Meeting, in: The Promise of the Herschel Space Observatory, p. 13.

Rayman, M.D., Lehman, D.H., 1997. Deep Space One: NASA's First DeepSpace Technology Validation Mission. Acta Astronautica 41, 289-299. doi:10.1016/S0094-5765(98)00073-3.

Ross, I.M., Fahroo, F., 2002. A Perspective on Methods for Trajectory Optimization, in: AIAA/AAS Astrodynamics Specialist Conference and Exhibit, p. 4727. doi:10.2514/6.2002-4727.

Ross, S.D., Scheeres, D.J., 2007. Multiple Gravity Assists, Capture, and Escape in the Restricted Three-Body Problem. SIAM Journal on Applied Dynamical Systems 6, 576-596. doi:10.1137/060663374.

Russell, C.T., Raymond, C.A., 2011. The Dawn Mission to Vesta and Ceres, in: The Dawn Mission to Minor Planets 4 Vesta and 1 Ceres. Springer, pp. 3-23. doi:10.1007/978-1-4614-4903-4_2. 
Sánchez, J.P., Neves, R., Urrutxua, H., 2018. Trajectory Design for Asteroid Retrieval Missions: A Short Review. Frontiers in Applied Mathematics and Statistics 4. doi:10.3389/fams.2018.00044.

Sánchez, J.P., Yárnoz, D.G., 2016. Asteroid Retrieval Missions enabled by Invariant Manifold Dynamics. Acta Astronautica 127, 667-677. doi:10. 1016/j . actaastro. 2016.05.034.

Shirazi, A., Ceberio, J., Lozano, J.A., 2018. Spacecraft Trajectory Optimization: A Review of Models, Objectives, Approaches and Solutions. Progress in Aerospace Sciences doi:10.1016/j.paerosci.2018.07.007.

Sims, J., Finlayson, P., Rinderle, E., Vavrina, M., Kowalkowski, T., 2006. Implementation of a Low-Thrust Trajectory Optimization Algorithm for Preliminary Design, in: AIAA/AAS Astrodynamics specialist conference and exhibit, p. 6746. doi:10.2514/6.2006-6746.

Sims, J., Flanagan, S., 1999. Preliminary Design of Low-Thrust Interplanetary Missions. Technical Report. NASA.

Szebehely, V., 1967. Theory of Orbits - The Restricted Problem of Three Bodies. Academic Press, London, UK.

Tauber, J.A., 2006. The Planck Mission, in: The Many Scales in the Universe. Springer, pp. 35-43. doi:10.1007/1-4020-4526-3_3.

Ulrich, W., 2019. Astronautics: the Physics of Space Flight. 3 ed., Springer. doi:10.1007/978-3-319-74373-8.

Yárnoz, D.G., Sánchez, J.P., McInnes, C., 2013. Easily Retrievable Objects among the NEO population. Celestial Mechanics and Dynamical Astronomy 116, 367-388. doi:10.1007/s10569-013-9495-6. 
2020-08-13

Low-thrust trajectory design in

low-energy regimes using variational equations

Neves, Rita

Elsevier

Neves R, Sánchez JP. (2020) Low-thrust trajectory design in low-energy regimes using

variational equations. Advances in Space Research, Volume 66, Issue 9, November 2020, pp. 2215-2231

https://doi.org/10.1016/j.asr.2020.07.047

Downloaded from Cranfield Library Services E-Repository 